\title{
SCHUR TYPE FUNCTIONS ASSOCIATED WITH POLYNOMIAL SEQUENCES OF BINOMIAL TYPE
}

\author{
MINORU ITOH
}

\begin{abstract}
We introduce a class of Schur type functions associated with polynomial sequences of binomial type. This can be regarded as a generalization of the ordinary Schur functions and the factorial Schur functions. This generalization satisfies some interesting expansion formulas, in which there is a curious duality. Moreover this class includes examples which are useful to describe the eigenvalues of Capelli type central elements of the universal enveloping algebras of classical Lie algebras.
\end{abstract}

\section{INTRODUCTION}

In this article, we introduce a class of Schur type functions associated with polynomial sequences of binomial type. Namely, inspired by the definition of the ordinary Schur function $\operatorname{det}\left(x_{j}^{\lambda_{i}+N-i}\right) / \operatorname{det}\left(x_{j}^{N-i}\right)$, we consider the following Schur type function:

$$
\operatorname{det}\left(p_{\lambda_{i}+N-i}\left(x_{j}\right)\right) / \operatorname{det}\left(p_{N-i}\left(x_{j}\right)\right) .
$$

Here $\left\{p_{n}(x)\right\}_{n \geq 0}$ is a polynomial sequence of binomial type. This can be regarded as a generalization of the ordinary Schur functions and of the factorial Schur functions ( $\mathrm{BL}$, [CL]). We also consider the following function:

$$
\operatorname{det}\left(p_{\lambda_{i}+N-i}^{*}\left(x_{j}\right)\right) / \operatorname{det}\left(p_{N-i}^{*}\left(x_{j}\right)\right) .
$$

Here we put $p_{n}^{*}(x)=x^{-1} p_{n+1}(x)$ (this is a polynomial, and satisfies good relations; see Section 1.3). The main results of this article are some expansion formulas for these functions and their mysterious duality corresponding to the exchange $p_{n}(x) \leftrightarrow p_{n}^{*}(x)$ and the conjugation of partitions (Sections 3-6). Most of them are proved by elementary and straightforward calculations. Besides these results, we also give an application to representation theory of Lie algebras (Section 8).

Let us briefly explain this application. The factorial Schur functions are useful to express the eigenvalues of Capelli type central elements of the universal enveloping algebras of the general linear Lie algebra (more precisely, we should say that the "shifted Schur functions" are useful; by the shift of variables, the factorial Schur functions are transformed into the shifted Schur functions ([OO1], [] $)$ ). In this article, we aim to introduce similar Schur type functions which is useful to express the eigenvalues of Capelli type central elements of the universal enveloping algebras of the orthogonal and symplectic Lie algebras. This aim is achieved in the case of the polynomial sequence corresponding to the central difference. This case associated with the central difference is also related with the analogues of the shifted Schur functions given in OO2], which were introduced

2000 Mathematics Subject Classification. Primary 05E05, 05A40; Secondary 17B35, 15A15;

Key words and phrases. Schur functions, polynomial sequence of binomial type, central elements of universal enveloping algebras.

This research was partially supported by JSPS Grant-in-Aid for Young Scientists (B) 17740080. 
with a similar aim. Moreover, from this investigation of eigenvalues, we see the relation between following two central elements of $U\left(\mathfrak{o}_{N}\right)$ and $U\left(\mathfrak{s p}_{N}\right)$ (Theorem 8.6): (a) the central elements in terms of the column-determinant and the column-permanent given in [W] and [16], (b) analogues of the quantum immanants given in OO2]. Our class is a natural generalization of the Schur functions containing these interesting functions related with the classical Lie algebras.

Various generalizations are known for the Schur functions. Many of them are obtained by replacing the ordinary powers by some polynomial sequence (further generalizations are known; see [M2]). In particular, the generalization associated with the polynomials in the form $p_{n}(x)=\prod_{k=1}^{n}\left(x-a_{k}\right)$ is well known [M1], and this contains the factorial Schur function. In this article, we consider another generalization which is not particularly large but includes interesting phenomena and examples.

\section{Polynomial sequences of Binomial type}

First, we recall the properties of polynomial sequences of binomial type. See $[M R],[R]$, [RKO], and [S] for further details.

1.1. We start with the definition. A polynomial sequence $\left\{p_{n}(x)\right\}_{n \geq 0}$ in which the degree of each polynomial is equal to its index is said to be of binomial type when the following relation holds for any $n \geq 0$ :

$$
p_{n}(x+y)=\sum_{k \geq 0}\left(\begin{array}{l}
n \\
k
\end{array}\right) p_{k}(x) p_{n-k}(y) .
$$

Let us see some examples. First, the sequence $\left\{x^{n}\right\}_{n \geq 0}$ of the ordinary powers is of binomial type, because we have the relation

$$
(x+y)^{n}=\sum_{k \geq 0}\left(\begin{array}{l}
n \\
k
\end{array}\right) x^{k} y^{n-k}
$$

(the ordinary binomial expansion). As other typical examples, some factorial powers are well known. We define the rising factorial power $x^{\bar{n}}$ and falling factorial power $x^{\underline{n}}$ by

$$
x^{\bar{n}}=x(x+1) \cdots(x+n-1), \quad x^{\underline{n}}=x(x-1) \cdots(x-n+1) .
$$

Then $\left\{x^{\bar{n}}\right\}_{n \geq 0}$ and $\left\{x^{\underline{n}}\right\}_{n \geq 0}$ are also of binomial type. Indeed the following relations hold ([MR], [RKO]):

$$
(x+y)^{\bar{n}}=\sum_{k \geq 0}\left(\begin{array}{l}
n \\
k
\end{array}\right) x^{\bar{k}} y^{\overline{n-k}}, \quad(x+y)^{\underline{n}}=\sum_{k \geq 0}\left(\begin{array}{l}
n \\
k
\end{array}\right) x^{\underline{k}} y^{\underline{n-k}} .
$$

It is easily seen that $p_{n}(0)=\delta_{n, 0}$, when $\left\{p_{n}(x)\right\}_{n \geq 0}$ is of binomial type.

1.2. A natural correspondence is known between polynomial sequences of binomial type and delta operators [RKO].

We recall the definition of delta operators. A linear operator $Q=Q_{x}: \mathbb{C}[x] \rightarrow \mathbb{C}[x]$ is called a delta operator when the following two properties hold: (i) $Q$ reduces degrees of polynomials by one; (ii) $Q$ is shift-invariant (that is, $Q$ commutes with all shift operators $\left.E^{a}: f(x) \mapsto f(x+a)\right)$. A typical example is the differentiation $D=\frac{d}{d x}$. Moreover the forward difference $\Delta^{+}$and the backward difference $\Delta^{-}$are delta operators:

$$
\Delta^{+}: f(x) \mapsto f(x+1)-f(x), \quad \Delta^{-}: f(x) \mapsto f(x)-f(x-1) .
$$


Every delta operator can be written as a power series in the differentiation operator $D$ of the following form with $a_{1}, a_{2}, \ldots \in \mathbb{C}, a_{1} \neq 0$ :

$$
Q=a_{1} D+a_{2} D^{2}+a_{3} D^{3}+\cdots .
$$

There is a natural one-to-one correspondence between these delta operators and polynomial sequences of binomial type. These are related via the relation

$$
Q p_{n}(x)=n p_{n-1}(x) .
$$

Namely, for a polynomial sequence of binomial type $\left\{p_{n}(x)\right\}_{n \geq 0}$, the linear operator $Q: \mathbb{C}[x] \rightarrow \mathbb{C}[x]$ determined by (1.1) is a delta operator. Conversely, for any delta operator $Q$, a polynomial sequence $\left\{p_{n}(x)\right\}_{n \geq 0}$ is uniquely determined by (1.1) and the relation $p_{n}(0)=\delta_{n, 0}$, and this sequence is of binomial type (these are called basic polynomials).

For example, the differentiation $D=\frac{d}{d x}$ corresponds to the sequence $\left\{x^{n}\right\}_{n \geq 0}$, because $D x^{n}=n x^{n-1}$. Similarly, the forward difference $\Delta^{+}$and the backward difference $\Delta^{-}$ correspond to the sequences $\left\{x^{\underline{n}}\right\}_{n \geq 0}$ and $\left\{x^{\bar{n}}\right\}_{n \geq 0}$, respectively:

$$
\Delta^{-} x^{\bar{n}}=n x^{\overline{n-1}}, \quad \Delta^{+} x^{\underline{n}}=n x^{\underline{n-1}} .
$$

1.3. There is another interesting polynomial sequence associated with a polynomial sequence of binomial type. For a polynomial sequence $\left\{p_{n}(x)\right\}_{n \geq 0}$ of binomial type, we put

$$
p_{n}^{*}(x)=x^{-1} p_{n+1}(x) .
$$

This is a polynomial, because the constant term of $p_{n+1}(x)$ is equal to 0 if $n \geq 0$ as seen above. This $p_{n}^{*}(x)$ satisfies the following relations (we can prove this by induction). In other words, $\left\{p_{n}^{*}(x)\right\}_{n \geq 0}$ is a Sheffer sequence $[\mathrm{R}]$.

Proposition 1.1. We have

$$
Q p_{n}^{*}(x)=n p_{n-1}^{*}(x), \quad p_{n}^{*}(x+y)=\sum_{k \geq 0}\left(\begin{array}{l}
n \\
k
\end{array}\right) p_{k}(x) p_{n-k}^{*}(y)=\sum_{k \geq 0}\left(\begin{array}{l}
n \\
k
\end{array}\right) p_{k}^{*}(x) p_{n-k}(y)
$$

These polynomials can be extended naturally for $n<0$ as elements of $\mathbb{C}\left(\left(x^{-1}\right)\right)=$ $\left\{\sum_{k \leq n} a_{k} x^{k} \mid a_{k} \in \mathbb{C}, n \in \mathbb{Z}\right\}$. Namely we have the following proposition (this is also proved by induction):

Proposition 1.2. Let $Q$ be a delta operator. Then there uniquely exist $\left\{p_{n}(x)\right\}_{n \in \mathbb{Z}}$ and $\left\{p_{n}^{*}(x)\right\}_{n \in \mathbb{Z}}$ satisfying the relations

$$
Q p_{n}(x)=n p_{n-1}(x), \quad Q p_{n}^{*}(x)=n p_{n-1}^{*}(x), \quad p_{n+1}(x)=x p_{n}^{*}(x)
$$

and

$$
\begin{aligned}
& p_{n}(x+y)=\sum_{k \geq 0}\left(\begin{array}{l}
n \\
k
\end{array}\right) p_{k}(x) p_{n-k}(y) \\
& p_{n}^{*}(x+y)=\sum_{k \geq 0}\left(\begin{array}{l}
n \\
k
\end{array}\right) p_{k}(x) p_{n-k}^{*}(y)=\sum_{k \geq 0}\left(\begin{array}{l}
n \\
k
\end{array}\right) p_{k}^{*}(x) p_{n-k}(y) .
\end{aligned}
$$

Here we regard the last two relations as equalities in $\mathbb{C}[x]\left(\left(y^{-1}\right)\right)$. 
Note that $p_{-1}^{*}(x)$ must be equal to $x^{-1}$, because $x p_{-1}^{*}(x)=p_{0}(x)=1$. Thus, this extension is unique.

From now on, we denote these polynomials associated with the delta operator $Q$ by $p_{n}(x)=p_{n}^{Q}(x)$ and $p_{n}^{*}(x)=p_{n}^{* Q}(x)$.

The polynomial $p_{n}^{*}(x)$ is not a mere supplementary object, but plays a role as important as $p_{n}$. We will exhibit some dualities between these two polynomials.

1.4. Consider the following operator:

$$
R_{x}=\left[Q_{x}, x\right]=Q_{x} x-x Q_{x}
$$

We can easily see that $R_{x} p_{k}^{*}(x)=p_{k}(x)$, and $R_{x}$ is invertible and shift-invariant. Let us put $p_{k}^{(a)}(x)=R_{x}^{a} p_{k}(x)$ for $a \in \mathbb{Z}$, so that $p_{k}^{(-1)}(x)=p_{k}^{*}(x)$. As seen by induction, this $p_{k}^{(a)}(x)$ satisfies the relation

$$
p_{n}^{(a+b)}(x+y)=\sum_{k \geq 0}\left(\begin{array}{l}
n \\
k
\end{array}\right) p_{n-k}^{(a)}(x) p_{k}^{(b)}(y) .
$$

In the case of $Q=\Delta^{+}$, this operator $R_{x}$ maps $f(x)$ to $f(x+1)$ (that is, $p_{n}^{(a)}(x)=$ $\left.(x+a-1)^{\underline{n}}\right)$, and this is an algebra automorphism on $\mathbb{C}[x]$, but this is not true for general $Q$. See also Remark in Section 2.

1.5. In the remainder of this article, we assume that $Q$ is normalized in the sense that the coefficient of $D$ is equal to 1 :

$$
Q=D+a_{2} D^{2}+a_{3} D^{3}+\cdots .
$$

Under this assumption, the associated polynomials $p_{n}(x)=p_{n}^{Q}(x)$ and $p_{n}^{*}(x)=p_{n}^{* Q}(x)$ become monic. Conversely, the delta operator associated with a monic polynomial sequence of binomial type is automatically normalized. Thus the following assumptions are equivalent: (i) $Q$ is normalized; (ii) $p_{n}^{Q}(x)$ is monic; (iii) $p_{n}^{* Q}(x)$ is monic. This assumption is not an essential one, but merely for simplicity.

1.6. Let us see some fundamental examples.

(1) In the case $Q=D=\frac{d}{d x}$, we have $p_{n}(x)=x^{n}$ and $p_{n}^{*}(x)=x^{n}$.

(2) In the case $Q=\Delta^{+}$, we have $p_{n}(x)=x^{\underline{n}}$, and hence $p_{n}^{*}(x)=(x-1)^{\underline{n}}$.

(3) In the case $Q=\Delta^{-}$, we have $p_{n}(x)=x^{\bar{n}}$, and hence $p_{n}^{*}(x)=(x+1)^{\bar{n}}$.

(4) We define the central difference $\Delta^{0}$ by

$$
\Delta^{0} f(x)=f\left(x+\frac{1}{2}\right)-f\left(x-\frac{1}{2}\right) .
$$

This is also a delta operator. In the case $Q=\Delta^{0}$, we have $p_{n}^{*}(x)=x^{\overline{\underline{n}}}$. Here we put

$$
x^{\underline{\underline{n}}}=\left(x+\frac{n-1}{2}\right)\left(x+\frac{n-3}{2}\right) \cdots\left(x-\frac{n-1}{2}\right) .
$$

Hence, $p_{n}(x)$ is expressed as $p_{n}(x)=x \cdot x^{\overline{\underline{n}}}$. This is seen by a direct calculation.

See $[\mathrm{R}]$, RKO], and [S] for other examples (Abel polynomials, Laguerre polynomials, etc.). 


\section{Definition of Schur type Functions}

Let us define our main objects, the Schur type functions associated with a polynomial sequence of binomial type. Let $p_{n}(x)=p_{n}^{Q}(x)$ and $p_{n}^{*}(x)=p_{n}^{* Q}(x)$ be polynomials corresponding to a normalized delta operator $Q$.

For $\lambda=\left(\lambda_{1}, \ldots, \lambda_{N}\right) \in \mathbb{Z}^{N}$, we consider the following determinants:

$$
\begin{gathered}
\tilde{s}_{\lambda}^{Q}\left(x_{1}, \ldots, x_{N}\right)=\operatorname{det}\left(\begin{array}{ccc}
p_{\lambda_{1}+N-1}\left(x_{1}\right) & \ldots & p_{\lambda_{1}+N-1}\left(x_{N}\right) \\
p_{\lambda_{2}+N-2}\left(x_{1}\right) & \ldots & p_{\lambda_{2}+N-2}\left(x_{N}\right) \\
\vdots & & \vdots \\
p_{\lambda_{N}+0}\left(x_{1}\right) & \ldots & p_{\lambda_{N}+0}\left(x_{N}\right)
\end{array}\right), \\
\tilde{s}_{\lambda}^{* Q}\left(x_{1}, \ldots, x_{N}\right)=\operatorname{det}\left(\begin{array}{ccc}
p_{\lambda_{1}+N-1}^{*}\left(x_{1}\right) & \ldots & p_{\lambda_{1}+N-1}^{*}\left(x_{N}\right) \\
p_{\lambda_{2}+N-2}^{*}\left(x_{1}\right) & \ldots & p_{\lambda_{2}+N-2}^{*}\left(x_{N}\right) \\
\vdots & & \vdots \\
p_{\lambda_{N}+0}^{*}\left(x_{1}\right) & \ldots & p_{\lambda_{N}+0}^{*}\left(x_{N}\right)
\end{array}\right) .
\end{gathered}
$$

We regard these as elements of

$$
\mathbb{C}\left(\left(x_{1}^{-1}, \ldots, x_{N}^{-1}\right)\right)=\left\{\sum_{k_{1} \leq n_{1}, \ldots, k_{N} \leq n_{N}} a_{k_{1}, \ldots, k_{N}} x_{1}^{k_{1}} \cdots x_{N}^{k_{N}} \mid a_{k_{1}, \ldots, k_{N}} \in \mathbb{C}, n_{1}, \ldots, n_{N} \in \mathbb{Z}\right\} .
$$

It is easy to see that these two determinants are alternating in $x_{1}, \ldots, x_{N}$. Moreover, when $\lambda=\emptyset=(0, \ldots, 0)$, these are equal to the difference product:

$$
\tilde{s}_{\emptyset}^{Q}\left(x_{1}, \ldots, x_{N}\right)=\tilde{s}_{\emptyset}^{* Q}\left(x_{1}, \ldots, x_{N}\right)=\Delta\left(x_{1}, \ldots, x_{N}\right)=\prod_{1 \leq i<j \leq N}\left(x_{i}-x_{j}\right) .
$$

Indeed, we can transform these to the ordinary Vandermonde determinant by elementary row operations, because $p_{n}(x)$ and $p_{n}^{*}(x)$ are monic. Having noted this, we consider the following functions:

$$
\begin{aligned}
s_{\lambda}^{Q}\left(x_{1}, \ldots, x_{N}\right) & =\tilde{s}_{\lambda}^{Q}\left(x_{1}, \ldots, x_{N}\right) / \tilde{s}_{\emptyset}^{Q}\left(x_{1}, \ldots, x_{N}\right), \\
s_{\lambda}^{* Q}\left(x_{1}, \ldots, x_{N}\right) & =\tilde{s}_{\lambda}^{* Q}\left(x_{1}, \ldots, x_{N}\right) / \tilde{s}_{\emptyset}^{* Q}\left(x_{1}, \ldots, x_{N}\right) .
\end{aligned}
$$

If $Q=D$, these are equal to the ordinary Schur functions. We can easily see that they are symmetric functions for any delta operator $Q$, and their highest degree parts are equal to the ordinary Schur function.

From now on, we omit the superscript $Q$, when it is clear from the context.

The functions $s_{\lambda}$ and $s_{\lambda}^{*}$ are polynomials, if $\lambda_{1}, \ldots, \lambda_{N} \geq 0$. When $\lambda=\left(\lambda_{1}, \ldots, \lambda_{N}\right)$ is a partition, namely when $\lambda_{1} \geq \cdots \geq \lambda_{N} \geq 0$, we can regard $\lambda$ as a Young diagram. In this case, the polynomials $s_{\lambda}$ (respectively, $s_{\lambda}^{*}$ ) form a basis of the space of symmetric polynomials.

We can also consider the counterparts of elementary symmetric functions and complete homogeneous symmetric functions (note that $h_{k}$ and $h_{k}^{*}$ can be defined even if $k$ is a 
negative integer):

$$
\begin{aligned}
e_{k}\left(x_{1}, \ldots, x_{N}\right) & =s_{\left(1^{k}\right)}\left(x_{1}, \ldots, x_{N}\right), \\
e_{k}^{*}\left(x_{1}, \ldots, x_{N}\right) & =s_{\left(1^{k}\right)}^{*}\left(x_{1}, \ldots, x_{N}\right), \\
h_{k}\left(x_{1}, \ldots, x_{N}\right) & =s_{(k)}\left(x_{1}, \ldots, x_{N}\right), \\
h_{k}^{*}\left(x_{1}, \ldots, x_{N}\right) & =s_{(k)}^{*}\left(x_{1}, \ldots, x_{N}\right) .
\end{aligned}
$$

Here we used the abbreviation

$$
\left(a_{1}^{m_{1}}, \ldots, a_{n}^{m_{n}}\right)=(\overbrace{a_{1}, \ldots, a_{1}}^{m_{1} \text { times }}, \ldots, \overbrace{a_{n}, \ldots, a_{n}}^{m_{n}}, 0, \ldots, 0) .
$$

These functions are not equal to the ordinary elementary symmetric functions and the ordinary complete symmetric functions in general, but there are two exceptions. Namely $e_{N}$ and $h_{-N}^{*}$ are independent of $\left\{p_{n}(x)\right\}$.

Proposition 2.1. We have

$$
e_{N}\left(x_{1}, \ldots, x_{N}\right)=x_{1} \cdots x_{N}, \quad h_{-N}^{*}\left(x_{1}, \ldots, x_{N}\right)=\frac{1}{x_{1} \cdots x_{N}} .
$$

This is easy from the following more general relation:

Proposition 2.2. We have

$$
s_{\left(\lambda_{1}, \ldots, \lambda_{N}\right)}\left(x_{1}, \ldots, x_{N}\right)=s_{\left(\lambda_{1}-1, \ldots, \lambda_{N}-1\right)}^{*}\left(x_{1}, \ldots, x_{N}\right) \cdot x_{1} \cdots x_{N} .
$$

This is immediate from

$$
\tilde{s}_{\left(\lambda_{1}, \ldots, \lambda_{N}\right)}\left(x_{1}, \ldots, x_{N}\right)=\tilde{s}_{\left(\lambda_{1}-1, \ldots, \lambda_{N}-1\right)}^{*}\left(x_{1}, \ldots, x_{N}\right) \cdot x_{1} \cdots x_{N}
$$

The following relation between $s$ and $s^{*}$ is also confirmed by a direct calculation:

Proposition 2.3. When $\lambda_{i} \geq 0$, we have

$$
s_{\left(\lambda_{1}, \ldots, \lambda_{N}\right)}^{*}\left(x_{1}, \ldots, x_{N}\right)=s_{\left(\lambda_{1}, \ldots, \lambda_{N}\right)}\left(x_{1}, \ldots, x_{N}, 0\right) .
$$

Remark. The shifted Schur function is defined as follows [01]:

$$
\operatorname{det}\left(\left(x_{j}+N-j\right) \frac{\lambda_{i}+N-i}{2}\right) / \operatorname{det}\left(\left(x_{j}+N-j\right) \frac{N-i}{}\right) .
$$

In the case of $Q_{x}=\Delta_{x}^{+}$, we have $p_{n}(x)=x^{\underline{n}}$, and $R_{x}=\left[Q_{x}, x\right]=Q_{x} x-x Q_{x}$ is equal to the algebra automorphism $f(x) \mapsto f(x+1)$. Thus this function can be expressed as

$$
\begin{aligned}
& \operatorname{det}\left(p_{\lambda_{i}+N-i}^{(N-j)}\left(x_{j}\right)\right) / \operatorname{det}\left(p_{N-i}^{(N-j)}\left(x_{j}\right)\right) \\
& \quad=R_{x_{1}}^{N-1} R_{x_{2}}^{N-2} \cdots R_{x_{N}}^{0} \operatorname{det}\left(p_{\lambda_{i}+N-i}\left(x_{j}\right)\right) / \operatorname{det}\left(p_{N-i}\left(x_{j}\right)\right) .
\end{aligned}
$$

Noting this and Proposition 2.3, we can naturally consider the projective limit of this function. This is an advantage of considering the shift $x_{j} \mapsto x_{j}+N-j$.

How about the case of an arbitrary delta operator $Q_{x}$ ? In general, we do not have such a good relation, because $R_{x}$ is not an algebra automorphism (thus this is not a polynomial in general, even if $\lambda$ is a partition). Thus it does not seem easy to consider a natural infinite-variable version for general $Q_{x}$. 


\section{ExpAnsions of Schur TyPE FUnCtions}

For the Schur type functions defined in the previous section, we have the following expansions (this can be regarded as a generalization of Example 10 in Section I.3 in [M1]):

Theorem 3.1. For $\lambda_{1} \geq \lambda_{2} \geq \cdots \geq \lambda_{N}$, we have

$$
\begin{aligned}
s_{\lambda}\left(x_{1}+u, \ldots, x_{N}+u\right) & =\sum_{\mu \subset \lambda} d_{\lambda \mu}(u) s_{\mu}\left(x_{1}, \ldots, x_{N}\right), \\
s_{\lambda}^{*}\left(x_{1}+u, \ldots, x_{N}+u\right) & =\sum_{\mu \subset \lambda} d_{\lambda \mu}(u) s_{\mu}^{*}\left(x_{1}, \ldots, x_{N}\right), \\
s_{\lambda}^{*}\left(x_{1}+u, \ldots, x_{N}+u\right) & =\sum_{\mu \subset \lambda} d_{\lambda \mu}^{*}(u) s_{\mu}\left(x_{1}, \ldots, x_{N}\right)
\end{aligned}
$$

as equalities in $\mathbb{C}[u]\left(\left(x_{1}^{-1}, \ldots, x_{N}^{-1}\right)\right)$. Here $\mu$ runs over $\mu=\left(\mu_{1}, \ldots, \mu_{N}\right)$ such that

$$
\mu_{1} \geq \mu_{2} \geq \cdots \geq \mu_{N}, \quad \mu_{1} \leq \lambda_{1}, \ldots, \mu_{N} \leq \lambda_{N},
$$

and $d_{\lambda \mu}(u)$ and $d_{\lambda \mu}^{*}(u)$ are defined by

$$
\begin{aligned}
& d_{\lambda \mu}(u)=\operatorname{det}\left(\left(\begin{array}{c}
\lambda_{i}+N-i \\
\lambda_{i}-\mu_{j}-i+j
\end{array}\right) p_{\lambda_{i}-\mu_{j}-i+j}(u)\right)_{1 \leq i, j \leq N} \\
& d_{\lambda \mu}^{*}(u)=\operatorname{det}\left(\left(\begin{array}{c}
\lambda_{i}+N-i \\
\lambda_{i}-\mu_{j}-i+j
\end{array}\right) p_{\lambda_{i}-\mu_{j}-i+j}^{*}(u)\right)_{1 \leq i, j \leq N}
\end{aligned}
$$

To prove this, we use the Cauchy-Binet formula:

Proposition 3.2. We have

$$
\operatorname{det}(A B)_{\left(i_{1}, \ldots, i_{k}\right),\left(j_{1}, \ldots, j_{k}\right)}=\sum_{1 \leq r_{1}<\cdots<r_{k}} \operatorname{det} A_{\left(i_{1}, \ldots, i_{k}\right),\left(r_{1}, \ldots, r_{k}\right)} \operatorname{det} B_{\left(r_{1}, \ldots, r_{k}\right),\left(j_{1}, \ldots, j_{k}\right)}
$$

Here we put $X_{\left(i_{1}, \ldots, i_{k}\right),\left(j_{1}, \ldots, j_{k}\right)}=\left(x_{i_{a}, j_{b}}\right)_{1 \leq a, b \leq k}$ for a matrix $X=\left(x_{i j}\right)$. Note that this holds when the multiplication is defined, even if the sizes of these matrices are infinite.

Proof of Theorem 3.1. We put $l_{i}=\lambda_{i}+N-i$, and consider the matrix

$$
A=\left(\begin{array}{ccc}
p_{l_{1}}\left(x_{1}+u\right) & \ldots & p_{l_{1}}\left(x_{N}+u\right) \\
\vdots & & \vdots \\
p_{l_{N}}\left(x_{1}+u\right) & \ldots & p_{l_{N}}\left(x_{N}+u\right)
\end{array}\right)
$$

The $(i, j)$ th entry $p_{l_{i}}\left(x_{j}+u\right)$ can be expanded as

$$
p_{l_{i}}\left(x_{j}+u\right)=\sum_{k \leq l_{1}}\left(\begin{array}{c}
l_{i} \\
l_{i}-k
\end{array}\right) p_{l_{i}-k}(u) p_{k}\left(x_{j}\right) .
$$


Thus $A$ is expressed as $A=B C$ with the $N \times \infty$ matrix $B$ and the $\infty \times N$ matrix $C$ defined by

$$
\begin{gathered}
B=\left(\begin{array}{ccc}
\left(\begin{array}{c}
l_{1} \\
0
\end{array}\right) p_{0}(u) & \left(\begin{array}{c}
l_{1} \\
1
\end{array}\right) p_{1}(u) & \ldots \\
\left(\begin{array}{c}
l_{2} \\
l_{2}-l_{1}
\end{array}\right) p_{l_{2}-l_{1}}(u) & \left(\begin{array}{c}
l_{2} \\
l_{2}-l_{1}+1
\end{array}\right) p_{l_{2}-l_{1}+1}(u) & \ldots \\
\vdots & \vdots & \\
\left(\begin{array}{c}
l_{N} \\
l_{N}-l_{1}
\end{array}\right) p_{l_{N}-l_{1}}(u) & \left(\begin{array}{c}
l_{N} \\
l_{N}-l_{1}+1
\end{array}\right) p_{l_{N}-l_{1}+1}(u) & \ldots
\end{array}\right), \\
C=\left(\begin{array}{cccc}
p_{l_{1}}\left(x_{1}\right) & p_{l_{1}}\left(x_{2}\right) & \ldots & p_{l_{1}}\left(x_{N}\right) \\
p_{l_{1}-1}\left(x_{1}\right) & p_{l_{1}-1}\left(x_{2}\right) & \ldots & p_{l_{1}-1}\left(x_{N}\right) \\
\vdots & \vdots & & \vdots
\end{array}\right) .
\end{gathered}
$$

Applying the Cauchy-Binet formula (Proposition 3.2) to this, we have

$$
\begin{aligned}
& \tilde{s}_{\lambda}\left(x_{1}+u, \ldots, x_{N}+u\right) \\
& =\operatorname{det} A
\end{aligned}
$$

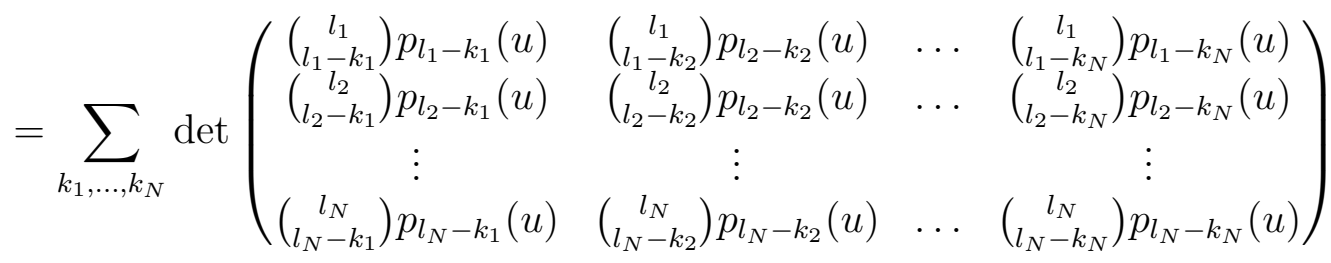

$$
\begin{aligned}
& \cdot \operatorname{det}\left(\begin{array}{cccc}
p_{k_{1}}\left(x_{1}\right) & p_{k_{1}}\left(x_{2}\right) & \ldots & p_{k_{1}}\left(x_{N}\right) \\
p_{k_{2}}\left(x_{1}\right) & p_{k_{2}}\left(x_{2}\right) & \ldots & p_{k_{2}}\left(x_{N}\right) \\
\vdots & \vdots & & \vdots \\
p_{k_{N}}\left(x_{1}\right) & p_{k_{N}}\left(x_{2}\right) & \ldots & p_{k_{N}}\left(x_{N}\right)
\end{array}\right) \\
& =\sum_{\mu \subset \lambda} d_{\lambda \mu}(u) \tilde{s}_{\mu}\left(x_{1}, \ldots, x_{N}\right) .
\end{aligned}
$$

Here, the first sum is over $k_{1}, \ldots, k_{N}$ satisfying $k_{i} \leq l_{i}$ and $k_{1}>\cdots>k_{N}$, and the second over $\mu_{1}, \ldots, \mu_{N}$ satisfying $\mu_{i} \leq \lambda_{i}$ and $\mu_{1} \geq \cdots \geq \mu_{N}$ (we define $\mu_{i}$ by $k_{i}=\mu_{i}+N-i$ ). Dividing this by $\Delta\left(x_{1}+u, \ldots, x_{N}+u\right)=\Delta\left(x_{1}, \ldots, x_{N}\right)$, we have the assertion.

It is interesting to consider the following variant of $d_{\lambda \mu}(u)$ when $\lambda$ and $\mu$ are partitions:

$$
\hat{d}_{\lambda \mu}(u)=\frac{\prod_{j}\left(\mu_{j}+N-j\right) !}{\prod_{i}\left(\lambda_{i}+N-i\right) !} d_{\lambda \mu}(u)=\operatorname{det}\left(p_{\left(\lambda_{i}-\mu_{j}-i+j\right)}(u)\right)_{1 \leq i, j \leq N} .
$$

Here we put $p_{(n)}(x)=\frac{1}{n !} p_{n}(x)$ suggested by the notation of divided power $x^{(n)}=\frac{1}{n !} x^{n}$. It is easily seen that $\hat{d}_{\lambda \mu}(u)$ is independent of $N$, though $d_{\lambda \mu}(u)$ depends on $N$. Namely, $\hat{d}_{\lambda \mu}$ does not change, even if we append some zeros at the ends of $\lambda$ and $\mu$. For this $\hat{d}_{\lambda \mu}(u)$, the following duality holds:

Theorem 3.3. For two partitions $\lambda$ and $\mu$, we have

$$
\hat{d}_{\lambda \mu}(u)=(-)^{|\lambda|-|\mu|} \hat{d}_{\lambda^{\prime} \mu^{\prime}}(-u) .
$$

Here $\lambda^{\prime}$ and $\mu^{\prime}$ mean the conjugates of $\lambda$ and $\mu$, respectively.

This theorem follows from Theorem 6.1 below. It can also be deduced from the following relation (essentially the same as (2.9) in [M1]), because $\sum_{k \geq 0} p_{(k)}(u) p_{(n-k)}(-u)=$ $p_{(n)}(0)=\delta_{n, 0}$ : 
Theorem 3.4. Assume that sequences $\left\{c_{k}\right\}_{k \geq 0}$ and $\left\{c_{k}^{\prime}\right\}_{k \geq 0}$ satisfy the relations $c_{0}=$ $c_{0}^{\prime}=1$ and $\sum_{k \geq 0}(-)^{k} c_{k} c_{n-k}^{\prime}=\delta_{n, 0}$. Then, for two partitions $\lambda$ and $\mu$, we have

$$
\operatorname{det}\left(c_{\lambda_{i}-\mu_{j}-i+j}\right)_{1 \leq i, j \leq \operatorname{depth} \lambda}=\operatorname{det}\left(c_{\lambda_{i}^{\prime}-\mu_{j}^{\prime}-i+j}\right)_{1 \leq i, j \leq \operatorname{depth} \lambda^{\prime}} .
$$

Here we interpret $c_{n}$ and $c_{n}^{\prime}$ as 0 for $n<0$.

\section{EXPANSIONS OF $e$ AND $h$}

The expansion formulas for the functions $e_{k}, e_{k}^{*}, h_{k}$, and $h_{k}^{*}$ have more interesting aspects. Some of these formulas are deduced from the results in the previous section as special cases, but the others are not, and we observe a mysterious duality in these formulas. For simplicity, we introduce the following notation:

$$
\begin{aligned}
h_{k}\left(x_{1}, \ldots, x_{N} ; u\right) & =h_{k}\left(x_{1}+u, \ldots, x_{N}+u\right), \\
h_{k}^{*}\left(x_{1}, \ldots, x_{N} ; u\right) & =h_{k}^{*}\left(x_{1}+u, \ldots, x_{N}+u\right), \\
e_{k}\left(x_{1}, \ldots, x_{N} ; u\right) & =e_{k}\left(x_{1}-u, \ldots, x_{N}-u\right), \\
e_{k}^{*}\left(x_{1}, \ldots, x_{N} ; u\right) & =e_{k}^{*}\left(x_{1}-u, \ldots, x_{N}-u\right) .
\end{aligned}
$$

These can be expanded as follows:

Theorem 4.1. For $k \geq 0$, we have

$$
\begin{aligned}
e_{k}\left(x_{1}, \ldots, x_{N} ; u\right) & =\sum_{l \geq 0}\left(\begin{array}{c}
-N+k-1 \\
k-l
\end{array}\right) e_{l}\left(x_{1}, \ldots, x_{N}\right) p_{k-l}(u) \\
& =\sum_{l \geq 0}\left(\begin{array}{c}
-N+k-1 \\
k-l
\end{array}\right) e_{l}^{*}\left(x_{1}, \ldots, x_{N}\right) p_{k-l}^{*}(u), \\
e_{k}^{*}\left(x_{1}, \ldots, x_{N} ; u\right) & =\sum_{l \geq 0}\left(\begin{array}{c}
-N+k-1 \\
k-l
\end{array}\right) e_{l}^{*}\left(x_{1}, \ldots, x_{N}\right) p_{k-l}(u) .
\end{aligned}
$$

Theorem 4.2. For $k \geq 0$, we have

$$
\begin{aligned}
h_{k}\left(x_{1}, \ldots, x_{N} ; u\right) & =\sum_{l \geq 0}\left(\begin{array}{c}
N+k-1 \\
k-l
\end{array}\right) h_{l}\left(x_{1}, \ldots, x_{N}\right) p_{k-l}(u), \\
h_{k}^{*}\left(x_{1}, \ldots, x_{N} ; u\right) & =\sum_{l \geq 0}\left(\begin{array}{c}
N+k-1 \\
k-l
\end{array}\right) h_{l}^{*}\left(x_{1}, \ldots, x_{N}\right) p_{k-l}(u) \\
& =\sum_{l \geq 0}\left(\begin{array}{c}
N+k-1 \\
k-l
\end{array}\right) h_{l}\left(x_{1}, \ldots, x_{N}\right) p_{k-l}^{*}(u) .
\end{aligned}
$$

Comparing these two theorems, we observe a duality corresponding to the exchanges $e \leftrightarrow h^{*}$ and $e^{*} \leftrightarrow h$. Note that the following are not equalities in general:

$$
\begin{aligned}
& e_{k}^{*}\left(x_{1}, \ldots, x_{N} ; u\right) \neq \sum_{l \geq 0}\left(\begin{array}{c}
-N+k-1 \\
k-l
\end{array}\right) e_{l}\left(x_{1}, \ldots, x_{N}\right) p_{k-l}^{*}(u), \\
& h_{k}\left(x_{1}, \ldots, x_{N} ; u\right) \neq \sum_{l \geq 0}\left(\begin{array}{c}
N+k-1 \\
k-l
\end{array}\right) h_{l}^{*}\left(x_{1}, \ldots, x_{N}\right) p_{k-l}^{*}(u) .
\end{aligned}
$$

Theorem 4.2 can be extended for negative integers $k$ as follows: 
Theorem 4.3. For $k \in \mathbb{Z}$, we have

$$
\begin{aligned}
h_{k}\left(x_{1}, \ldots, x_{N} ; u\right) & =\sum_{l \geq 0}\left(\begin{array}{c}
N+k-1 \\
N+l-1
\end{array}\right) h_{l}\left(x_{1}, \ldots, x_{N}\right) p_{k-l}(u), \\
h_{k}^{*}\left(x_{1}, \ldots, x_{N} ; u\right) & =\sum_{l \geq 0}\left(\begin{array}{c}
N+k-1 \\
N+l-1
\end{array}\right) h_{l}^{*}\left(x_{1}, \ldots, x_{N}\right) p_{k-l}(u) \\
& =\sum_{l \geq 0}\left(\begin{array}{c}
N+k-1 \\
N+l-1
\end{array}\right) h_{l}\left(x_{1}, \ldots, x_{N}\right) p_{k-l}^{*}(u) .
\end{aligned}
$$

Theorem 4.4. For $k \in \mathbb{Z}$, we have

$$
\begin{aligned}
h_{k}\left(x_{1}, \ldots, x_{N} ; u\right) & =\sum_{l \geq 0}\left(\begin{array}{c}
N+k-1 \\
l
\end{array}\right) h_{k-l}\left(x_{1}, \ldots, x_{N}\right) p_{l}(u), \\
h_{k}^{*}\left(x_{1}, \ldots, x_{N} ; u\right) & =\sum_{l \geq 0}\left(\begin{array}{c}
N+k-1 \\
l
\end{array}\right) h_{k-l}^{*}\left(x_{1}, \ldots, x_{N}\right) p_{l}(u) \\
& =\sum_{l \geq 0}\left(\begin{array}{c}
N+k-1 \\
l
\end{array}\right) h_{k-l}\left(x_{1}, \ldots, x_{N}\right) p_{l}^{*}(u) .
\end{aligned}
$$

We can prove Theorems 4.24.4 easily in a way similar to the general expansion Theorem 3.1. The proof of Theorem 4.1 is as follows:

Proof of Theorem 4.1. First we prove the case of $k=N$. Noting (2.1), we have

$$
\begin{aligned}
& \tilde{s}_{\left(1^{N}\right)}\left(x_{1}-u, \ldots, x_{N}-u\right) \\
& =\operatorname{det}\left(\begin{array}{ccc}
p_{N}\left(x_{1}-u\right) & \ldots & p_{N}\left(x_{N}-u\right) \\
\vdots & & \vdots \\
p_{1}\left(x_{1}-u\right) & \ldots & p_{1}\left(x_{N}-u\right)
\end{array}\right) \\
& =\left(x_{1}-u\right) \cdots\left(x_{N}-u\right) \operatorname{det}\left(\begin{array}{ccc}
p_{N-1}^{*}\left(x_{1}-u\right) & \ldots & p_{N-1}^{*}\left(x_{N}-u\right) \\
\vdots & & \vdots \\
p_{0}^{*}\left(x_{1}-u\right) & \ldots & p_{0}^{*}\left(x_{N}-u\right)
\end{array}\right) \\
& =\left(x_{1}-u\right) \cdots\left(x_{N}-u\right) \Delta\left(x_{1}-u, \ldots, x_{N}-u\right) \\
& =\left(x_{1}-u\right) \cdots\left(x_{N}-u\right) \Delta\left(x_{1}, \ldots, x_{N}\right) \\
& =\Delta\left(x_{1}, \ldots, x_{N}, u\right) \\
& =\operatorname{det}\left(\begin{array}{cccc}
p_{N}\left(x_{1}\right) & \ldots & p_{N}\left(x_{N}\right) & p_{N}(u) \\
\vdots & & \vdots & \vdots \\
p_{0}\left(x_{1}\right) & \ldots & p_{0}\left(x_{N}\right) & p_{0}(u)
\end{array}\right)
\end{aligned}
$$

By the cofactor expansion along the last column, we see that this is equal to

$$
\sum_{k=0}^{N}(-)^{k} \tilde{s}_{\left(1^{N-k}\right)}\left(x_{1}, \ldots, x_{N}\right) p_{k}(u) .
$$


Dividing both sides by the difference product $\Delta\left(x_{1}-u, \ldots, x_{N}-u\right)=\Delta\left(x_{1}, \ldots, x_{N}\right)$, we have

$$
e_{N}\left(x_{1}-u, \ldots, x_{N}-u\right)=\sum_{k \geq 0}(-)^{k} p_{k}(u) e_{N-k}\left(x_{1}, \ldots, x_{N}\right) .
$$

Thus we have the assertion for $k=N$.

The other cases are deduced from this. Indeed, on one hand, we have

$$
e_{N}\left(x_{1}-u-w, \ldots, x_{N}-u-w\right)=\sum_{l \geq 0}(-)^{l} p_{l}(w) e_{N-l}\left(x_{1}-u, \ldots, x_{N}-u\right),
$$

and on the other hand

$$
\begin{aligned}
e_{N}\left(x_{1}-u-w, \ldots, x_{N}-u-w\right) & =\sum_{k \geq 0}(-)^{k} p_{k}(u+w) e_{N-k}\left(x_{1}, \ldots, x_{N}\right) \\
& =\sum_{k \geq 0} \sum_{l \geq 0}(-)^{k}\left(\begin{array}{c}
k \\
l
\end{array}\right) p_{l}(w) p_{k-l}(u) e_{N-k}\left(x_{1}, \ldots, x_{N}\right) .
\end{aligned}
$$

Comparing the coefficients of $p_{l}(w)$, we have the general case.

The following relation for the delta operator is easy to deduce from these expansions:

Corollary 4.5. We have

$$
\begin{aligned}
& Q_{u} h_{k}\left(x_{1}, \ldots, x_{N} ; u\right)=(N+k-1) h_{k-1}\left(x_{1}, \ldots, x_{N} ; u\right), \\
& Q_{u} h_{k}^{*}\left(x_{1}, \ldots, x_{N} ; u\right)=(N+k-1) h_{k-1}^{*}\left(x_{1}, \ldots, x_{N} ; u\right), \\
& Q_{u} e_{k}\left(x_{1}, \ldots, x_{N} ; u\right)=(-N+k-1) e_{k-1}\left(x_{1}, \ldots, x_{N} ; u\right), \\
& Q_{u} e_{k}^{*}\left(x_{1}, \ldots, x_{N} ; u\right)=(-N+k-1) e_{k-1}^{*}\left(x_{1}, \ldots, x_{N} ; u\right) .
\end{aligned}
$$

\section{Generating functions}

Combining Proposition 2.1 with the relations in the previous section, we have the following relations (put $k=N$ in Theorem 4.1 and $k=-N$ in Theorems 4.3 and 4.4):

Theorem 5.1. We have

$$
\begin{aligned}
\left(u-x_{1}\right) \cdots\left(u-x_{N}\right) & =\sum_{l \geq 0}(-)^{l} e_{l}\left(x_{1}, \ldots, x_{N}\right) p_{N-l}(u) \\
& =\sum_{l \geq 0}(-)^{l} e_{l}^{*}\left(x_{1}, \ldots, x_{N}\right) p_{N-l}^{*}(u) .
\end{aligned}
$$

Theorem 5.2. We have

$$
\begin{aligned}
\frac{1}{\left(u+x_{1}\right) \cdots\left(u+x_{N}\right)} & =\sum_{l \geq 0}(-)^{l} h_{l}\left(x_{1}, \ldots, x_{N}\right) p_{-N-l}^{*}(u) \\
& =\sum_{l \geq 0}(-)^{l} h_{l}^{*}\left(x_{1}, \ldots, x_{N}\right) p_{-N-l}(u) .
\end{aligned}
$$

Theorem 5.3. We have

$$
\begin{aligned}
\frac{1}{\left(u+x_{1}\right) \cdots\left(u+x_{N}\right)} & =(-)^{N-1} \sum_{l \geq 0}(-)^{l} h_{-N-l}\left(x_{1}, \ldots, x_{N}\right) p_{l}^{*}(u) \\
& =(-)^{N-1} \sum_{l \geq 0}(-)^{l} h_{-N-l}^{*}\left(x_{1}, \ldots, x_{N}\right) p_{l}(u) .
\end{aligned}
$$


We can regard the left hand sides of these equalities as "generating functions" of $e_{k}$, $e_{k}^{*}, h_{k}$, and $h_{k}^{*}$ represented as sums of multiples of $p_{n}(x)$ or $p_{n}^{*}(x)$ instead of the ordinary power.

Remark. The conclusion of Theorem 5.1 also holds, even if $\left\{p_{n}(u)\right\}$ is not of binomial type. Namely, if $p_{n}(u)$ is a monic polynomial of degree $n$, we have

$$
\left(u-x_{1}\right) \cdots\left(u-x_{N}\right)=\sum_{l \geq 0}(-)^{l} e_{l}\left(x_{1}, \ldots, x_{N}\right) p_{N-l}(u) .
$$

This is easily seen from the proof of Theorem 4.1. Similarly, the assertions of Theorems 5.2 and 5.3 also hold, if $p_{n}(x)$ and $p_{n}^{*}(x)$ are monic polynomials of degree $n$ satisfying the relation

$$
\frac{1}{x+y}=\sum_{k \geq 0}(-)^{k} p_{k}(x) p_{-1-k}^{*}(y)=\sum_{k \geq 0}(-)^{k} p_{k}^{*}(x) p_{-1-k}(y) .
$$

\section{Cauchy type Relations}

The relations in the previous section can be generalized as analogues of the (dual) Cauchy identity. In this section, we often abbreviate a function $f\left(x_{1}, \ldots, x_{N}\right)$ simply as $f(x)$.

Theorem 6.1. We have

$$
\prod_{1 \leq i \leq M} \prod_{1 \leq j \leq N}\left(y_{j}-x_{i}\right)=\sum_{\lambda}(-)^{|\lambda|} s_{\lambda}(x) s_{\lambda^{\dagger}}(y)=\sum_{\lambda}(-)^{|\lambda|} s_{\lambda}^{*}(x) s_{\lambda^{\dagger}}^{*}(y) .
$$

Here $\lambda$ runs over the Young diagrams satisfying $\operatorname{depth}(\lambda) \leq M$ and $\operatorname{depth}\left(\lambda^{\prime}\right) \leq N$. Moreover we define $\lambda^{\dagger}$ by

$$
\lambda^{\dagger}=\left(N-\lambda_{M}, N-\lambda_{M-1}, \ldots, N-\lambda_{1}\right)^{\prime} .
$$

Theorem 6.2. We have

$$
\prod_{1 \leq i \leq M} \prod_{1 \leq j \leq N} \frac{1}{y_{j}+x_{i}}=\sum_{\lambda}(-)^{|\lambda|} s_{\lambda}^{*}(x) s_{\lambda^{\ddagger}}(y)=\sum_{\lambda}(-)^{|\lambda|} s_{\lambda}(x) s_{\lambda^{\ddagger}}^{*}(y)
$$

in $\mathbb{C}\left[x_{1}, \ldots, x_{M}\right]\left(\left(y_{1}^{-1}, \ldots, y_{N}^{-1}\right)\right)$. Here $\lambda$ runs over the Young diagrams $\lambda$ satisfying $\operatorname{depth}(\lambda) \leq \min (M, N)$. Moreover we define $\lambda^{\ddagger}$ by

$$
\lambda^{\ddagger}=\left(-M-\lambda_{N},-M-\lambda_{N-1}, \ldots,-M-\lambda_{1}\right) .
$$

From Theorem 6.1 we see the following duality:

$$
(-)^{|\lambda|} d_{\lambda \mu}(u)=(-)^{|\mu|} d_{\mu^{\dagger} \lambda^{\dagger}}(-u) \text {. }
$$

Indeed, this follows by expanding $\prod_{1 \leq i \leq M} \prod_{1 \leq j \leq N} \frac{1}{y_{j}+x_{i}+u}$ in two ways. Theorem 3.3 is immediate from this.

Remark. As in the previous section, the conclusion of Theorem 6.1 holds even if $\left\{p_{n}(u)\right\}$ is not of binomial type. Namely it holds if $p_{n}(u)$ is a monic polynomial of degree $n$. Similarly the conclusion of Theorem 6.2 also holds if $p_{n}(x)$ and $p_{n}^{*}(x)$ are monic polynomials of degree $n$ satisfying

$$
\frac{1}{x+y}=\sum_{k \geq 0}(-)^{k} p_{k}(x) p_{-1-k}^{*}(y)=\sum_{k \geq 0}(-)^{k} p_{k}^{*}(x) p_{-1-k}(y) .
$$


Remark. We can regard Theorem 6.2 as a generalization of the following well-known relation (the Cauchy identity):

$$
\prod_{1 \leq i \leq M, 1 \leq i \leq N} \frac{1}{1-x_{i} y_{j}}=\sum_{\lambda} s_{\lambda}^{D}(x) s_{\lambda}^{D}(y) .
$$

Proof of Theorem 6.1. By (2.1) we have

$$
\begin{aligned}
& \Delta(y) \Delta(x) \prod_{1 \leq i \leq N} \prod_{1 \leq j \leq M}\left(y_{j}-x_{i}\right)=\Delta\left(y_{1}, \ldots, y_{N}, x_{1}, \ldots, x_{M}\right) \\
&=\operatorname{det}\left(\begin{array}{cccccc}
p_{M+N-1}\left(y_{1}\right) & \ldots & p_{M+N-1}\left(y_{N}\right) & p_{M+N-1}\left(x_{1}\right) & \ldots & p_{M+N-1}\left(x_{M}\right) \\
p_{M+N-2}\left(y_{1}\right) & \ldots & p_{M+N-2}\left(y_{N}\right) & p_{M+N-2}\left(x_{1}\right) & \ldots & p_{M+N-2}\left(x_{M}\right) \\
\vdots & & \vdots & \vdots & & \vdots \\
p_{0}\left(y_{1}\right) & \ldots & p_{0}\left(y_{N}\right) & p_{0}\left(x_{1}\right) & \ldots & p_{0}\left(x_{M}\right)
\end{array}\right) .
\end{aligned}
$$

Applying the Laplace expansion to the first $N$ columns, we see that this is equal to $\sum_{\lambda}(-)^{|\lambda|} \tilde{s}_{\lambda}(x) \tilde{s}_{\lambda^{\dagger}}(y)$. Indeed we have

$$
\left\{\lambda_{i}+M-i \mid 1 \leq i \leq M\right\} \cup\left\{\lambda_{j}^{\dagger}+N-j \mid 1 \leq j \leq N\right\}=\{0,1, \ldots, M+N-1\}
$$

(recall (1.7) in [M1]). This means the first equality. The second equality is similarly shown by replacing $p_{k}$ by $p_{k}^{*}$.

To prove Theorem 6.2, we use the following well-known relation (the left hand side is known as the Cauchy determinant):

Lemma 6.3. When $M=N$, we have

$$
\operatorname{det}\left(\frac{1}{x_{i}+y_{j}}\right)=\frac{\Delta(x) \Delta(y)}{\prod_{1 \leq i, j \leq N}\left(x_{i}+y_{j}\right)} .
$$

This relation is generalized as follows (this can be proved by induction):

Lemma 6.4. When $N \geq M$, we have

$$
\operatorname{det}\left(\begin{array}{cccc}
p_{N-M-1}^{*}\left(y_{1}\right) & p_{N-M-1}^{*}\left(y_{2}\right) & \ldots & p_{N-M-1}^{*}\left(y_{N}\right) \\
\vdots & \vdots & & \vdots \\
p_{1}^{*}\left(y_{1}\right) & p_{1}^{*}\left(y_{2}\right) & \ldots & p_{1}^{*}\left(y_{N}\right) \\
p_{0}^{*}\left(y_{1}\right) & p_{0}^{*}\left(y_{2}\right) & \ldots & p_{0}^{*}\left(y_{N}\right) \\
\frac{1}{x_{1}+y_{1}} & \frac{1}{x_{1}+y_{2}} & \ldots & \frac{1}{x_{1}+y_{N}} \\
\frac{1}{x_{2}+y_{1}} & \frac{1}{x_{2}+y_{2}} & \ldots & \frac{1}{x_{2}+y_{N}} \\
\vdots & \vdots & & \vdots \\
\frac{1}{x_{M}+y_{1}} & \frac{1}{x_{M}+y_{2}} & \ldots & \frac{1}{x_{M}+y_{N}}
\end{array}\right)=\frac{\Delta(x) \Delta(y)}{\prod_{1 \leq i \leq M, 1 \leq j \leq N}\left(x_{i}+y_{j}\right)} .
$$

Proof of Theorem 6.2 (the case of $M=N$ ). Using the Cauchy-Binet formula (Proposition 3.2), we have

$$
\begin{aligned}
& \operatorname{det}\left(\frac{1}{x_{i}+y_{j}}\right)_{1 \leq i, j \leq N} \\
& \quad=\operatorname{det}\left(\sum_{k \geq 0}(-)^{k} p_{k}\left(x_{i}\right) p_{-1-k}^{*}\left(y_{j}\right)\right)_{1 \leq i, j \leq N}
\end{aligned}
$$




$$
\begin{aligned}
& =\sum_{0 \leq k_{1}<\cdots<k_{N}} \operatorname{det}\left((-)^{k_{i}} p_{k_{i}}\left(x_{j}\right)\right)_{1 \leq i, j \leq N} \operatorname{det}\left(p_{-1-k_{i}}^{*}\left(y_{j}\right)\right)_{1 \leq i, j \leq N} \\
& =\sum_{0 \leq k_{1}<\cdots<k_{N}}(-)^{k_{1}+\cdots+k_{N}} \operatorname{det}\left(p_{k_{i}}\left(x_{j}\right)\right)_{1 \leq i, j \leq N} \operatorname{det}\left(p_{-1-k_{i}}^{*}\left(y_{j}\right)\right)_{1 \leq i, j \leq N} .
\end{aligned}
$$

Here, the first determinant on the right hand side is equal to

$$
\operatorname{det}\left(p_{\lambda_{i}+N-i}\left(x_{j}\right)\right)_{1 \leq i, j \leq N}=\tilde{s}_{\lambda}(x),
$$

where we define $\lambda_{i}$ by

$$
k_{N}=\lambda_{1}+N-1, \quad k_{N-1}=\lambda_{2}+N-2, \quad \ldots, \quad k_{1}=\lambda_{N}+0 .
$$

On the other hand, the second determinant is equal to

$$
\operatorname{det}\left(p_{-1-N-\lambda_{i}+i}^{*}\left(y_{j}\right)\right)_{1 \leq i, j \leq N}=\tilde{s}_{\lambda^{\ddagger}}^{*}(y) .
$$

Thus we have

$$
\operatorname{det}\left(\frac{1}{x_{i}+y_{j}}\right)_{1 \leq i, j \leq N}=\sum_{\lambda}(-)^{|\lambda|} \tilde{s}_{\lambda}(x) \tilde{s}_{\lambda^{\ddagger}}^{*}(y),
$$

and the assertion is immediate by dividing this by $\Delta(x) \Delta(y)$.

Proof of Theorem 6.2 (the case of $N>M$ ). Let us denote by $A$ the matrix on the left hand side of Lemma 6.4. This can be expressed as

$$
A=\left(\begin{array}{ccc}
p_{N-M-1}^{*}\left(y_{1}\right) & \ldots & p_{N-M-1}^{*}\left(y_{N}\right) \\
\vdots & & \vdots \\
p_{0}^{*}\left(y_{1}\right) & \ldots & p_{0}^{*}\left(y_{N}\right) \\
\sum_{k}(-)^{k} p_{k}\left(x_{1}\right) p_{-1-k}^{*}\left(y_{1}\right) & \ldots & \sum_{k}(-)^{k} p_{k}\left(x_{1}\right) p_{-1-k}^{*}\left(y_{N}\right) \\
\vdots & & \vdots \\
\sum_{k}(-)^{k} p_{k}\left(x_{M}\right) p_{-1-k}^{*}\left(y_{1}\right) & \ldots & \sum_{k}(-)^{k} p_{k}\left(x_{M}\right) p_{-1-k}^{*}\left(y_{N}\right)
\end{array}\right)=B C
$$

with the $N \times \infty$ matrix $B$ and the $\infty \times N$ matrix $C$ defined by

$$
\begin{aligned}
B & =\left(\begin{array}{cccccc}
1 & \ldots & 0 & 0 & 0 & \ldots \\
\vdots & \ddots & \vdots & \vdots & \vdots & \\
0 & \ldots & 1 & 0 & 0 & \ldots \\
0 & \ldots & 0 & (-)^{0} p_{0}\left(x_{1}\right) & (-)^{1} p_{1}\left(x_{1}\right) & \ldots \\
\vdots & & \vdots & \vdots & \vdots & \\
0 & \ldots & 0 & (-)^{0} p_{0}\left(x_{M}\right) & (-)^{1} p_{1}\left(x_{M}\right) & \ldots
\end{array}\right) \\
C & =\left(\begin{array}{ccc}
p_{N-M-1}^{*}\left(y_{1}\right) & \ldots & p_{N-M-1}^{*}\left(y_{N}\right) \\
p_{N-M-2}^{*}\left(y_{1}\right) & \ldots & p_{N-M-2}^{*}\left(y_{N}\right) \\
\vdots & & \vdots
\end{array}\right) .
\end{aligned}
$$

Applying the Cauchy-Binet formula (Proposition 3.2) to this relation $A=B C$, we have

$$
\operatorname{det} A=\operatorname{det} B C=\sum_{1 \leq i_{1}<\cdots<i_{N}} \operatorname{det} B_{(1, \ldots, N),\left(i_{1}, \ldots, i_{N}\right)} \operatorname{det} C_{\left(i_{1}, \ldots, i_{N}\right),(1, \ldots, N)} .
$$


Note that $\operatorname{det} B_{(1, \ldots, N),\left(i_{1}, \ldots, i_{N}\right)}=0$, unless $\left(i_{1}, \ldots, i_{N-M}\right)=(1, \ldots, N-M)$. Thus we have

$$
\begin{gathered}
\operatorname{det} A=\sum_{0 \leq k_{1}<\cdots<k_{M}} \operatorname{det}\left(\begin{array}{cccccc}
1 & \ldots & 0 & 0 & \ldots & 0 \\
\vdots & \ddots & \vdots & \vdots & & \vdots \\
0 & \ldots & 1 & 0 & \ldots & 0 \\
0 & \ldots & 0 & (-)^{k_{1}} p_{k_{1}}\left(x_{1}\right) & \ldots & (-)^{k_{M}} p_{k_{M}}\left(x_{1}\right) \\
\vdots & & \vdots & \vdots & & \vdots \\
0 & \ldots & 0 & (-)^{k_{1}} p_{k_{1}}\left(x_{M}\right) & \ldots & (-)^{k_{M}} p_{k_{M}}\left(x_{M}\right)
\end{array}\right) \\
\cdot \operatorname{det}\left(\begin{array}{cccc}
p_{N-M-1}^{*}\left(y_{1}\right) & \ldots & p_{N-M-1}^{*}\left(y_{N}\right) \\
\vdots & & & \vdots \\
p_{0}^{*}\left(y_{1}\right) & \ldots & p_{0}^{*}\left(y_{N}\right) \\
p_{-1-k_{1}}^{*}\left(y_{1}\right) & \ldots & p_{-1-k_{1}}^{*}\left(y_{N}\right) \\
\vdots & & \vdots \\
p_{-1-k_{M}}^{*}\left(y_{1}\right) & \ldots & p_{-1-k_{M}}^{*}\left(y_{N}\right)
\end{array}\right) .
\end{gathered}
$$

The first determinant is equal to

$$
\begin{aligned}
\operatorname{det}\left(\begin{array}{ccc}
(-)^{k_{1}} p_{k_{1}}\left(x_{1}\right) & \ldots & (-)^{k_{M}} p_{k_{M}}\left(x_{1}\right) \\
\vdots & \vdots & \vdots \\
(-)^{k_{1}} p_{k_{1}}\left(x_{M}\right) & \ldots & (-)^{k_{M}} p_{k_{M}}\left(x_{M}\right)
\end{array}\right) \\
\quad=(-)^{k_{1}+\cdots+k_{M}} \operatorname{det}\left(\begin{array}{cccc}
p_{k_{1}}\left(x_{1}\right) & \ldots & p_{k_{M}}\left(x_{1}\right) \\
\vdots & & \vdots \\
p_{k_{1}}\left(x_{M}\right) & \ldots & p_{k_{M}}\left(x_{M}\right)
\end{array}\right) \\
=(-)^{k_{1}+\cdots+k_{M}}(-)^{\frac{M(M-1)}{2}} \operatorname{det}\left(\begin{array}{ccc}
p_{k_{M}}\left(x_{1}\right) & \ldots & p_{k_{1}}\left(x_{1}\right) \\
\vdots & & \vdots \\
p_{k_{M}}\left(x_{M}\right) & \ldots & p_{k_{1}}\left(x_{M}\right)
\end{array}\right) \\
=(-)^{\lambda_{1}+\cdots+\lambda_{M}} \operatorname{det}\left(\begin{array}{ccc}
p_{\lambda_{1}+M-1}\left(x_{1}\right) & \ldots & p_{\lambda_{M}}\left(x_{1}\right) \\
\vdots & & \vdots \\
p_{\lambda_{1}+M-1}\left(x_{M}\right) & \ldots & p_{\lambda_{M}}\left(x_{M}\right)
\end{array}\right) \\
=(-)^{|\lambda|} \tilde{s}_{\lambda}(x) .
\end{aligned}
$$

Here we define $\lambda_{1}, \ldots, \lambda_{M}$ by

$$
k_{M}=\lambda_{1}+M-1, \quad k_{M-1}=\lambda_{2}+M-2, \quad \ldots, \quad k_{1}=\lambda_{M}+0 .
$$

The second determinant in the formula for $\operatorname{det} A$ is equal to

$$
\operatorname{det}\left(\begin{array}{ccc}
p_{\lambda_{1}^{\ddagger}+N-1}^{*}\left(y_{1}\right) & \ldots & p_{\lambda_{1}^{\ddagger}+N-1}^{*}\left(y_{N}\right) \\
p_{\lambda_{2}^{\ddagger}+N-2}^{*}\left(y_{1}\right) & \ldots & p_{\lambda_{2}^{\ddagger}+N-2}^{*}\left(y_{N}\right) \\
\vdots & & \vdots \\
p_{\lambda_{N}^{\ddagger}+0}^{*}\left(y_{1}\right) & \ldots & p_{\lambda_{N}^{\ddagger}+0}^{*}\left(y_{N}\right)
\end{array}\right)=\tilde{s}_{\lambda^{\ddagger}}^{*}(y) .
$$

Here we put

$$
\lambda_{1}^{\ddagger}=-M-\lambda_{M}, \quad \lambda_{2}^{\ddagger}=-M-\lambda_{M-1}, \quad \ldots, \quad \lambda_{N}^{\ddagger}=-M-\lambda_{1} .
$$


Thus we have

$$
\operatorname{det} A=\sum_{\lambda}(-)^{|\lambda|} \tilde{s}_{\lambda}(x) \tilde{s}_{\lambda^{\ddagger}}^{*}(y) .
$$

This yields our assertion.

The proof in the case $N<M$ is almost the same, so we omit it.

\section{Capelli type elements}

Our Schur type functions are useful to express the eigenvalues of Capelli type central elements of the universal enveloping algebras of the classical Lie algebras. Before stating this, we recall these Capelli type elements in this section.

These central elements have been investigated in the study of Capelli type identities. See [HU], [MN], O], [U1-5], [IU], and [I1-6] for the Capelli identity and its generalizations.

7.1. First, we recall the Capelli elements, famous central elements of the universal enveloping algebra $U\left(\mathfrak{g l}_{N}\right)$ of the general linear Lie algebra $\mathfrak{g l}_{N}$. Let $E_{i j}$ be the standard basis of $\mathfrak{g l}_{N}$, and consider the matrix $E=\left(E_{i j}\right)_{1 \leq i, j \leq N}$ in $\operatorname{Mat}_{N}\left(U\left(\mathfrak{g l}_{N}\right)\right)$. Then the following determinant is known as the Capelli element ([Ca1], [HU], [U1]):

$$
C^{\mathfrak{g l}_{N}}(u)=\operatorname{det}\left(E-u \mathbf{1}+\operatorname{diag} \natural_{N}\right) .
$$

Here 1 is the unit matrix, and $\bigsqcup_{N}$ is the sequence $\bigsqcup_{N}=(N-1, N-2, \ldots, 0)$ of length $N$. Moreover "det" means a non-commutative determinant called the column-determinant. Namely, for a square matrix $Z=\left(Z_{i j}\right)$ whose entries are non-commutative, we put

$$
\operatorname{det} Z=\sum_{\sigma \in \mathfrak{S}_{N}} \operatorname{sgn}(\sigma) Z_{\sigma(1) 1} Z_{\sigma(2) 2} \cdots Z_{\sigma(N) N} .
$$

The Capelli element $C^{\mathfrak{g l}_{N}}(u)$ is known to be central in $U\left(\mathfrak{g l}_{N}\right)$.

This is generalized to the sums of minors

$$
C_{k}^{\mathfrak{g l}_{N}}(u)=\sum_{1 \leq i_{1}<\cdots<i_{k} \leq N} \operatorname{det}\left(E_{I}-u \mathbf{1}+\operatorname{diag} \natural_{k}\right) .
$$

Here we put $Z_{I}=\left(Z_{i_{a} i_{b}}\right)_{1 \leq a, b \leq k}$ for $I=\left(i_{1}, \ldots, i_{k}\right)$ and $Z=\left(Z_{i j}\right)$. We call this the Capelli element of degree $k$.

Moreover we can consider the following analogue using the permanent $[\mathrm{N}]$ :

$$
D_{k}^{\mathfrak{g l}_{N}}(u)=\sum_{1 \leq i_{1} \leq \cdots \leq i_{k} \leq N} \frac{1}{I !} \operatorname{per}\left(E_{I}+u \mathbf{1}_{I}-\mathbf{1}_{I} \operatorname{diag} \natural_{k}\right) .
$$

Here "per" means the column-permanent. Namely, for a square matrix $Z=\left(Z_{i j}\right)$ of size $N$, we put

$$
\text { per } Z=\sum_{\sigma \in \mathfrak{S}_{N}} Z_{\sigma(1) 1} \cdots Z_{\sigma(N) N}
$$

Here we put $I !=m_{1} ! \cdots m_{N} !$, where $m_{1}, \ldots, m_{N}$ are the multiplicities of $I=\left(i_{1}, \ldots, i_{k}\right)$ :

$$
I=\left(i_{1}, \ldots, i_{k}\right)=(\overbrace{1, \ldots, 1}^{m_{1}}, \overbrace{2, \ldots, 2}^{m_{2}}, \ldots, \overbrace{N, \ldots, N}^{m_{N}}) .
$$

Since $I$ has some multiplicities in general, $Z_{I}=\left(Z_{i_{a} i_{b}}\right)_{1 \leq a, b \leq k}$ is not a submatrix of $Z$ necessarily. 
The elements $C_{k}^{\mathfrak{g l}_{N}}(u)$ and $D_{k}^{\mathfrak{g l}_{N}}(u)$ are also central in the universal enveloping algebra (actually these are generators of the center of the universal enveloping algebra; see [HU], [I4], [N], [U1] for the details).

Theorem 7.1. For any $u \in \mathbb{C}, C_{k}^{\mathfrak{g l}_{N}}(u)$ and $D_{k}^{\mathfrak{g l}_{N}}(u)$ are central in $U\left(\mathfrak{g l}_{N}\right)$.

These central elements act on the irreducible representations as scalar operators by Schur's lemma. These values (the eigenvalues) can be calculated by noting the following triangular decomposition:

$$
\mathfrak{g l}_{N}=\mathfrak{n}^{-} \oplus \mathfrak{h} \oplus \mathfrak{n}^{+} .
$$

Here $\mathfrak{n}^{-}, \mathfrak{h}$, and $\mathfrak{n}^{+}$are the subalgebras of $\mathfrak{g l}_{N}$ spanned by the elements $F_{i j}^{\mathfrak{g l}_{N}}$ such that $i>j, i=j$, and $i<j$, respectively. Namely, the entries in the lower triangular part, in the diagonal part, and in the upper triangular part of the matrix $E^{\mathfrak{g l}_{N}}$ belong to $\mathfrak{n}^{-}, \mathfrak{h}$, and $\mathfrak{n}^{+}$, respectively.

For example, we can calculate the eigenvalue of $C^{\mathfrak{g l}_{N}}(u)$ as follows. Let $\pi$ be the irreducible representation determined by the partition $\left(\lambda_{1}, \ldots, \lambda_{N}\right)$, and consider the action of $C^{\mathfrak{g l}_{N}}(u)$ to the highest weight vector $v$ :

$$
\pi\left(C^{\mathfrak{g l}_{N}}(u)\right) v=\sum_{\sigma \in \mathfrak{S}_{N}} \operatorname{sgn}(\sigma) \pi\left(E_{\sigma(1) 1}(-u+N-1)\right) \cdots \pi\left(E_{\sigma(N) N}(-u+0)\right) v .
$$

Then, among these $N$ ! terms, there remains only one term corresponding to $\sigma=\left(\begin{array}{cccc}1 & 2 & \ldots & N \\ 1 & 2 & \ldots & N\end{array}\right)$, and the other $N$ ! -1 terms all vanish, because $\pi\left(E_{i j}\right) v=0$ for $i<j$ and $\pi\left(E_{i i}\right) v=\lambda_{i} v$. Thus we have

$$
\begin{aligned}
\pi\left(C^{\mathfrak{g l}_{N}}(u)\right) v & =\pi\left(E_{11}(-u+N-1)\right) \cdots \pi\left(E_{N N}(-u+0)\right) v \\
& =\left(\lambda_{1}-u+N-1\right) \cdots\left(\lambda_{N}-u+0\right) v .
\end{aligned}
$$

and we see that $C^{\mathfrak{g l}_{N}}(u)$ acts as multiplication by the scalar $\left(\lambda_{1}-u+N-1\right) \cdots\left(\lambda_{N}-u+0\right)$ on $\pi$.

We can write down the eigenvalue of $C_{k}^{\mathfrak{g l}_{N}}(u)$ similarly by considering the action to the highest weight vector $v$ of each column-determinant on the right hand side of (7.1). Indeed, also in this case, only one term remains of the $k$ ! terms in each column-determinant.

The calculation of the eigenvalue of $D_{k}^{\mathfrak{g l}_{N}}(u)$ is essentially same (but a bit more complicated). For this, we consider the action of each column-permanent on the right hand side of (7.2) to $v$. Then, of the $k$ ! terms in each column-permanent, there remain only $I$ ! terms corresponding to $\sigma$ such that $i_{\sigma(a)}=i_{a}$ for $a=1, \ldots, k$, and these $I$ ! terms are all equal to a scalar multiple of $v$.

In this way, we can write down the eigenvalues of $C_{k}^{\mathfrak{g l}_{N}}(u)$ and $D_{k}^{\mathfrak{g l}_{N}}(u)$. However the results are not so simple. As seen in Section 8 below, these eigenvalues are actually expressed by using the factorial (shifted) Schur functions.

Remark. We note some previous results:

(1) We can also express the elements $C_{k}^{\mathfrak{g l}_{N}}(u)$ and $D_{k}^{\mathfrak{g l}_{N}}(u)$ in terms of the "symmetrized determinant" and the "symmetrized permanents" ([IU], [I1-6]). From these expressions, we can easily see the centrality of these elements.

(2) In [O], Okounkov introduced a class of central elements of $U\left(\mathfrak{g l}_{N}\right)$, and called them the quantum immanants. These elements $\mathbb{S}_{\mu}$ indexed by partitions $\mu$ are expressed in terms of a determinant type function called immanant, and form a basis of the center of $U\left(\mathfrak{g l}_{N}\right)$ 
as a vector. The central elements $C_{k}^{\mathfrak{g l}_{N}}(0)$ and $D_{k}^{\mathfrak{g l}_{N}}(0)$ can be regarded as the cases of $\mu=\left(1^{k}\right)$ and $\mu=(k)$ :

$$
C_{k}^{\mathfrak{g l}}(0)=\mathbb{S}_{\left(1^{k}\right)}, \quad D_{k}^{\mathfrak{g l}}(0)=\mathbb{S}_{(k)} .
$$

Okounkov also gave a generalization of the Capelli identity (called higher Capelli identities) for the quantum immanants. In Section 8 , we will see the eigenvalues of the quantum immanants together with those of $C_{k}^{\mathfrak{g l}_{N}}(u)$ and $D_{k}^{\mathfrak{g l}_{N}}(u)$.

7.2. Next, we recall analogues of the Capelli elements in the universal enveloping algebras of the orthogonal Lie algebras given in [W].

Let $S \in \operatorname{Mat}_{N}(\mathbb{C})$ be a non-singular symmetric matrix of size $N$. We can realize the orthogonal Lie group as the isometry group with respect to the bilinear form determined by $S$ :

$$
O(S)=\left\{g \in G L_{N} \mid{ }^{t} g S g=S\right\} .
$$

The corresponding Lie algebra is expressed as

$$
\mathfrak{o}(S)=\left\{\left.Z \in \mathfrak{g l}_{N}\right|^{t} Z S+S Z=0\right\} .
$$

As generators of this $\mathfrak{o}(S)$, we can take $F_{i j}^{\mathfrak{o}(S)}=E_{i j}-S^{-1} E_{j i} S$, where $E_{i j}$ is the standard basis of $\mathfrak{g l}_{N}$. We introduce the $N \times N$ matrix $F^{\mathfrak{o}(S)}$ whose $(i, j)$ th entry is this generator: $F^{\mathfrak{o}(S)}=\left(F_{i j}^{\mathfrak{o}(S)}\right)_{1 \leq i, j \leq N}$. We regard this matrix as an element of $\operatorname{Mat}_{N}(U(\mathfrak{o}(S)))$.

In particular, in the case of $S=S_{0}=\left(\delta_{i, N+1-j}\right)$, the corresponding orthogonal Lie algebra is expressed as follows:

$$
\mathfrak{o}\left(S_{0}\right)=\left\{Z=\left(Z_{i j}\right) \in \mathfrak{g l}_{N} \mid Z_{i j}+Z_{N+1-j, N+1-i}=0\right\} .
$$

We call this the split realization of the orthogonal Lie algebra. In this case, we can take the following triangular decomposition:

$$
\mathfrak{o}\left(S_{0}\right)=\mathfrak{n}^{-} \oplus \mathfrak{h} \oplus \mathfrak{n}^{+} .
$$

Here $\mathfrak{n}^{-}, \mathfrak{h}$, and $\mathfrak{n}^{+}$are the subalgebras of $\mathfrak{o}\left(S_{0}\right)$ spanned by the elements $F_{i j}^{\mathfrak{o}\left(S_{0}\right)}$ such that $i>j, i=j$, and $i<j$, respectively. Namely, the entries in the lower triangular part, in the diagonal part, and in the upper triangular part of the matrix $E^{\mathfrak{g l}_{N}}$ belong to $\mathfrak{n}^{-}, \mathfrak{h}$, and $\mathfrak{n}^{+}$, respectively. Thus, if there is a central element of $U\left(\mathfrak{o}\left(S_{0}\right)\right)$ which is expressed as the column-determinant of $F^{\mathfrak{o}\left(S_{0}\right)}$, we can easily calculate its eigenvalue in a way similar to the case of $C^{\mathfrak{g l}_{N}}(u)$. In fact, the following central element was given by Wachi [W]:

Theorem 7.2 (Wachi). For any $u \in \mathbb{C}$, the following element is central in $U\left(\mathfrak{o}\left(S_{0}\right)\right)$ :

$$
C^{\mathfrak{o}_{N}}(u)=\operatorname{det}\left(F^{\mathfrak{o}\left(S_{0}\right)}-u \mathbf{1}+\operatorname{diag} \tilde{\natural}_{N}\right) .
$$

Here $\tilde{\natural}_{N}$ is the following sequence of length $N$ :

$$
\tilde{\natural}_{N}= \begin{cases}\left(\frac{N}{2}-1, \frac{N}{2}-2, \ldots, 0,0, \ldots,-\frac{N}{2}+1\right), & N: \text { even, } \\ \left(\frac{N}{2}-1, \frac{N}{2}-2, \ldots, \frac{1}{2}, 0,-\frac{1}{2}, \ldots,-\frac{N}{2}+1\right), & N: \text { odd. }\end{cases}
$$

This can be generalized as follows:

Theorem 7.3 (Wachi). For any $u \in \mathbb{C}$, the following element is central in $U\left(\mathfrak{o}\left(S_{0}\right)\right)$ :

$$
C_{k}^{\mathfrak{o}_{N}}(u)=\sum_{1 \leq i_{1}<\cdots<i_{k} \leq N} \operatorname{det}\left(\widetilde{F}_{I}^{\mathfrak{o}\left(S_{0}\right)}-u \mathbf{1}+\operatorname{diag}\left(\frac{k}{2}-1, \frac{k}{2}-2, \ldots,-\frac{k}{2}\right)\right) .
$$


Here $\widetilde{F}^{\mathfrak{o}\left(S_{0}\right)}$ is defined by

$$
\widetilde{F}^{\mathfrak{o}\left(S_{0}\right)}= \begin{cases}F^{\mathfrak{o}\left(S_{0}\right)}+\operatorname{diag}(0, \ldots, 0,1, \ldots, 1), & N: \text { even, } \\ F^{\mathfrak{o}\left(S_{0}\right)}+\operatorname{diag}\left(0, \ldots, 0, \frac{1}{2}, 1, \ldots, 1\right), & N: \text { odd } .\end{cases}
$$

Here the numbers of 0 's and 1's are equal to $[N / 2]$.

In Section 8, we will express the eigenvalues of these central elements in terms of the Schur type functions associated with the central difference.

7.3. Similarly, we can construct central elements in the universal enveloping algebra of the symplectic Lie algebra. However, these are expressed in terms of permanents (not in terms of determinants).

Let $J \in \operatorname{Mat}_{N}(\mathbb{C})$ be a non-singular alternating matrix of size $N$. We can realize the symplectic Lie group as the isometry group with respect to the bilinear form determined by $J$ :

$$
S p(J)=\left\{g \in G L_{N} \mid{ }^{t} g J g=J\right\} .
$$

The corresponding Lie algebra is expressed as

$$
\mathfrak{s p}(J)=\left\{\left.Z \in \mathfrak{g l}_{N}\right|^{t} Z J+J Z=0\right\} .
$$

As generators of this $\mathfrak{s p}(J)$, we can take $F_{i j}^{\mathfrak{s p}(J)}=E_{i j}-J^{-1} E_{j i} J$, where $E_{i j}$ is the standard basis of $\mathfrak{g l}_{N}$. We introduce the $N \times N$ matrix $F^{\mathfrak{s p}(J)}$ whose $(i, j)$ th entry is this generator: $F^{\mathfrak{s p}(J)}=\left(F_{i j}^{\mathfrak{s p}(J)}\right)_{1 \leq i, j \leq N}$. We regard this matrix as an element of $\operatorname{Mat}_{N}(U(\mathfrak{s p}(J)))$.

We consider the split realization of the symplectic Lie algebra. Namely we consider the case of

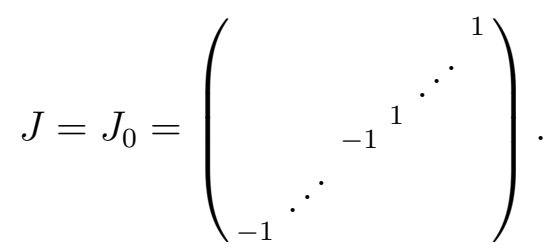

In this case, we can take the following triangular decomposition:

$$
\mathfrak{s p}\left(J_{0}\right)=\mathfrak{n}^{-} \oplus \mathfrak{h} \oplus \mathfrak{n}^{+} .
$$

Here $\mathfrak{n}^{-}, \mathfrak{h}$, and $\mathfrak{n}^{+}$are the subalgebras of $\mathfrak{s p}\left(J_{0}\right)$ spanned by the elements $F_{i j}^{\mathfrak{s p}\left(J_{0}\right)}$ such that $i>j, i=j$, and $i<j$, respectively. Namely, the entries in the lower triangular part, in the diagonal part, and in the upper triangular part of the matrix $E^{\mathfrak{g l}_{N}}$ belong to $\mathfrak{n}^{-}$, $\mathfrak{h}$, and $\mathfrak{n}^{+}$, respectively. In this case, we can construct central elements of the universal enveloping algebra using the column-permanent [I6]:

Theorem 7.4 (Itoh). For any $u \in \mathbb{C}$, the following element is central in $U\left(\mathfrak{s p}\left(J_{0}\right)\right)$ :

$$
D_{k}^{\mathfrak{s p}}(u)=\sum_{1 \leq i_{1} \leq \cdots \leq i_{k} \leq N} \frac{1}{I !} \operatorname{per}\left(\widetilde{F}_{I}^{\mathfrak{s p}\left(J_{0}\right)}+u \mathbf{1}_{I}-\mathbf{1}_{I} \operatorname{diag}\left(\frac{k}{2}-1, \frac{k}{2}-2, \ldots,-\frac{k}{2}\right)\right) .
$$

Here we put

$$
\widetilde{F}^{\mathfrak{s p}\left(J_{0}\right)}=F^{\mathfrak{s p}\left(J_{0}\right)}-\operatorname{diag}(0, \ldots, 0,1, \ldots, 1),
$$

where the numbers of 0 's and 1's are equal to $N / 2$.

We can easily calculate the eigenvalue of this central element noting the triangular decomposition of $\mathfrak{s p}\left(J_{0}\right)$ and the definition of the column-permanent. In Section 8, we will express them in terms of the Schur type functions associated with the central difference. 
Remark. We note some previous results:

(1) We can also express $C_{k}^{\mathfrak{o}_{N}}(0)$ and $D_{k}^{\mathfrak{s p}_{N}}(0)$ in terms of the symmetrized determinant and the symmetrized permanent ([W], [I5], [I6]). Capelli type identities in terms of these symmetrized determinant and permanent are also given in [I3] and [I4].

(2) Analogues of the quantum immanants in $U\left(\mathfrak{o}_{N}\right)$ and $U\left(\mathfrak{s p}_{N}\right)$ are studied in $\mathrm{OO} 2$. We will see in Section 8 that these can be regarded as a generalization of $C_{k}^{\mathfrak{o}_{N}}(0)$ and $D_{k}^{\mathfrak{s p}_{N}}(0)$.

\section{The eigenvalues of CAPElli type Elements}

Finally we consider concrete examples of Schur type functions associated with some differences. When $Q$ is the forward difference, the associated Schur type functions are equal to the factorial Schur functions. By the shift of variables, these are transformed into the shifted Schur functions, and these are useful to express the eigenvalues of some central elements of the universal enveloping algebra of the general linear Lie algebra. When $Q$ is the central difference, the associated Schur functions are useful to express the eigenvalues of the central elements of the universal enveloping algebras of the orthogonal and symplectic Lie algebras listed in the previous section.

8.1. Let us consider the case that $Q$ is equal to the forward difference $\Delta^{+}$. In this case, $p_{n}^{\Delta^{+}}(x)$ and $p_{n}^{* \Delta^{+}}(x)$ are expressed as $p_{n}^{\Delta^{+}}(x)=x^{\underline{n}}$ and $p_{n}^{* \Delta^{+}}(x)=(x-1) \underline{n}$. The corresponding symmetric functions $e_{k}^{\Delta^{+}}$and $h_{k}^{\Delta^{+}}$are explicitly expressed as follows $\left(e_{k}^{* \Delta^{+}}\right.$ and $h_{k}^{* \Delta^{+}}$are also given by considering the shift of variables). This expression is essentially equivalent with Corollary 11.3 in [OO1].

Theorem 8.1. We have

$$
\begin{aligned}
& e_{k}^{\Delta^{+}}\left(x_{1}, \ldots, x_{N}\right) \\
& \quad=\sum_{1 \leq i_{1}<\cdots<i_{k} \leq N}\left(x_{i_{1}}-N+k-1+i_{1}\right)\left(x_{i_{2}}-N+k-2+i_{2}\right) \cdots\left(x_{i_{k}}-N+i_{k}\right), \\
& h_{k}^{\Delta^{+}}\left(x_{1}, \ldots, x_{N}\right) \\
& \quad=\sum_{1 \leq i_{1} \leq \cdots \leq i_{k} \leq N}\left(x_{i_{1}}-N-k+1+i_{1}\right)\left(x_{i_{2}}-N-k+2+i_{2}\right) \cdots\left(x_{i_{k}}-N+i_{k}\right) .
\end{aligned}
$$

Proof. The first relation is obtained from the relation

$$
e_{N}\left(x_{1}-u, \ldots, x_{N}-u\right)=\left(x_{1}-u\right) \cdots\left(x_{N}-u\right) .
$$

It suffices to apply $Q_{u}$ repeatedly and use the Leibnitz rule for the forward difference: $\Delta^{+}(f(x) g(x))=\Delta^{+} f(x) g(x+1)+f(x) \Delta^{+} g(x)$. The second relation is deduced by induction on $N$ (use Theorems 5.2 and 5.3 ).

Using these symmetric functions, we can describe the eigenvalues of $C_{k}^{\mathfrak{g l}_{N}}(u)$ and $D_{k}^{\mathfrak{g l}_{N}}(u)$ defined in the previous section as follows:

Theorem 8.2. For the representation $\pi_{\lambda}^{\mathfrak{g l}_{N}}$ of $\mathfrak{g l}_{N}$ determined by the partition $\lambda=$ $\left(\lambda_{1}, \ldots, \lambda_{N}\right)$, we have

$$
\pi_{\lambda}^{\mathfrak{g l}_{N}}\left(C_{k}^{\mathfrak{g l}_{N}}(u)\right)=e_{k}^{\Delta^{+}}\left(l_{1}, \ldots, l_{N} ; u\right), \quad \pi_{\lambda}^{\mathfrak{g l}_{N}}\left(D_{k}^{\mathfrak{g l}_{N}}(u)\right)=h_{k}^{\Delta^{+}}\left(l_{1}, \ldots, l_{N} ; u\right) .
$$

Here we put $l_{i}=\lambda_{i}+N-i$. 
We can deduce this by the procedure outlined in Section 7 using the triangular decomposition of the general linear Lie algebra and the definitions of the column-determinant and the column-permanent.

Theorem 8.2 is essentially included in the following description of the eigenvalues of the quantum immanants in terms of the factorial (shifted) Schur functions due to [001]:

$$
\pi_{\lambda}^{\mathfrak{g l}_{N}}\left(\mathbb{S}_{\mu}\right)=s_{\mu}^{\Delta^{+}}\left(l_{1}, \ldots, l_{N}\right) .
$$

Indeed, when $u=0$, Theorem 8.2 is a special case of this relation as seen from (7.3). Moreover, the case of $u \neq 0$ can be also deduced from this by considering the algebra automorphisms $E_{i j} \mapsto E_{i j}+u \delta_{i j}$ and $E_{i j} \mapsto E_{i j}-u \delta_{i j}$ on $U\left(\mathfrak{g l}_{N}\right)$.

8.2. Next, let us consider the case of the central difference (namely $Q=\Delta^{0}$ ). In this case, $p_{n}^{\Delta^{0}}(x)$ and $p_{n}^{* \Delta^{0}}(x)$ are expressed as $p_{n}^{\Delta^{0}}(x)=x \cdot x \underline{\overline{n-1}}$ and $p_{n}^{* \Delta^{0}}(x)=x^{\overline{\underline{n}}}$. Moreover $e_{k}^{\Delta^{0}}$ and $h_{k}^{* \Delta^{0}}$ are expressed as follows:

Theorem 8.3. We have

$$
\begin{aligned}
e_{k}^{\Delta^{0}} & \left(x_{1}, \ldots, x_{N}\right) \\
& =\sum_{1 \leq i_{1}<\cdots<i_{k} \leq N}\left(x_{i_{1}}-\frac{N}{2}+\frac{k}{2}-1+i_{1}\right)\left(x_{i_{2}}-\frac{N}{2}+\frac{k}{2}-2+i_{2}\right) \cdots\left(x_{i_{k}}-\frac{N}{2}-\frac{k}{2}+i_{k}\right) \\
& =\sum_{1 \leq i_{1}<\cdots<i_{k} \leq N}\left(x_{i_{1}}+\frac{N}{2}-\frac{k}{2}+1-i_{1}\right)\left(x_{i_{2}}+\frac{N}{2}-\frac{k}{2}+2-i_{2}\right) \cdots\left(x_{i_{k}}+\frac{N}{2}+\frac{k}{2}-i_{k}\right), \\
h_{k}^{* \Delta^{0}} & \left(x_{1}, \ldots, x_{N}\right) \\
& =\sum_{1 \leq i_{1} \leq \cdots \leq i_{k} \leq N}\left(x_{i_{1}}-\frac{N}{2}-\frac{k}{2}+i_{1}\right)\left(x_{i_{2}}-\frac{N}{2}-\frac{k}{2}+1+i_{2}\right) \cdots\left(x_{i_{k}}-\frac{N}{2}+\frac{k}{2}-1+i_{k}\right) \\
& =\sum_{1 \leq i_{1} \leq \cdots \leq i_{k} \leq N}\left(x_{i_{1}}+\frac{N}{2}+\frac{k}{2}-i_{1}\right)\left(x_{i_{2}}+\frac{N}{2}+\frac{k}{2}-1-i_{2}\right) \cdots\left(x_{i_{k}}+\frac{N}{2}-\frac{k}{2}+1-i_{k}\right) .
\end{aligned}
$$

The proof of the first relation is almost the same as that of Theomem 8.1 . Here, we use the Leibnitz rule for the central difference

$$
\Delta^{0}(f(x) g(x))=\Delta^{0} f(x) g\left(x+\frac{1}{2}\right)+f\left(x-\frac{1}{2}\right) \Delta^{0} g(x) .
$$

The second relation is deduced from the second formula in Theomem 8.1 by replacing $x_{i}$ with $x_{i}^{\prime}=x_{i}+\frac{N}{2}+\frac{k}{2}$. Indeed, using elementary row operations, we have

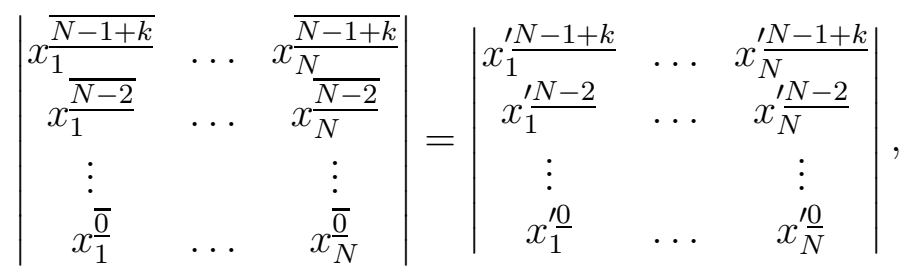

and this means the relation

$$
\tilde{s}_{(k)}^{* \Delta^{0}}\left(x_{1}, \ldots, x_{N}\right)=\tilde{s}_{(k)}^{\Delta^{+}}\left(x_{1}^{\prime}, \ldots, x_{N}^{\prime}\right) .
$$

Using these functions $e_{k}^{\Delta^{0}}$ and $h_{k}^{* \Delta^{0}}$, we can express the eigenvalues of the Capelli type central elements of the universal enveloping algebras of the orthogonal and symplectic Lie algebras.

First, we have the following relation for $C_{k}^{\mathfrak{o}_{N}}(u)$ : 
Theorem 8.4. For the irreducible representation $\pi_{\lambda}^{\mathfrak{o}_{N}}$ of $\mathfrak{o}_{N}$ determined by the partition $\lambda=\left(\lambda_{1}, \ldots, \lambda_{[n]}\right)$, we have

$$
\pi_{\lambda}^{\mathfrak{o}_{N}}\left(C_{k}^{\mathfrak{o}_{N}}(u)\right)=e_{k}^{\Delta^{0}}\left(l_{1}, \ldots, l_{N} ; u\right) .
$$

Here we define $l_{1}, \ldots, l_{N}$ as follows. First, for $1 \leq i \leq n$, we put $l_{i}=\lambda_{i}+n-i$ (that is, we consider the $\rho$-shift). Next, we put $l_{n+1}=-l_{n}, \ldots, l_{N}=-l_{1}$ when $N$ is even, and we put $l_{n^{\dagger}}=0, l_{n^{\dagger}+1}=-l_{n^{\dagger}-1}, \ldots, l_{N}=-l_{1}$ with $n^{\dagger}=\frac{N+1}{2}$ when $N$ is odd.

Similarly we have the following relation for $D_{k}^{\mathfrak{s p}_{N}}(u)$ :

Theorem 8.5. For the irreducible representation $\pi_{\lambda}^{\mathfrak{s p}_{N}}$ of $\mathfrak{s p}_{N}$ determined by the partition $\lambda=\left(\lambda_{1}, \ldots, \lambda_{n}\right)$, we have

$$
\pi_{\lambda}^{\mathfrak{s p}_{N}}\left(D_{k}^{\mathfrak{s p}}(u)\right)=h_{k}^{* \Delta^{0}}\left(l_{1}, \ldots, l_{N} ; u\right)
$$

Here $l_{i}$ is defined as follows: we put $l_{i}=\lambda_{i}+n+1-i$ for $1 \leq i \leq n$ (that is, we consider the $\rho$-shift), and put $l_{n+1}=-l_{n}, \ldots, l_{N}=-l_{1}$ for $n+1 \leq i \leq N$.

Remark. Factorial powers and differences were key tools in the study of Capelli type elements, and various relations for them have been given ([I1-6], [IU], and [U1-5]). The formulas in Sections 4 and 5 of this article can be regarded as natural generalizations of these relations.

8.3. The functions $e_{k}^{\Delta^{0}}$ and $h_{k}^{* \Delta^{0}}$ are related with analogues of the shifted Schur functions due to Okounkov and Olshanski. Moreover, from these relations and the calculation of the eigenvalues in Theorems 8.4 and 8.5, we see that $C_{k}^{\mathfrak{o}_{N}}(0)$ and $D_{k}^{\mathfrak{s p}_{N}}(0)$ are equal to special cases of analogues of the quantum immanants. Let us see these relations.

In [O2], Okounkov and Olshanski introduced analogues of the quantum immanants in the universal enveloping algebras of the simple Lie algebras of types B, C, and D, and denoted these elements by $\mathbb{T}_{\mu}$. Let us write these as $\mathbb{T}_{\mu}^{B}, \mathbb{T}_{\mu}^{C}$, and $\mathbb{T}_{\mu}^{D}$, when we need to indicate the type of the Lie algebra. Noting that the eigenvalues of these central elements are polynomials in $l_{1}^{2}, \ldots, l_{n}^{2}$, Okounkov and Olshanski also defined a class of functions $t_{\mu}^{*}$ corresponding to the types $\mathrm{B}, \mathrm{C}$, and $\mathrm{D}$ by the relation

$$
\pi_{\lambda}\left(\mathbb{T}_{\mu}\right)=t_{\mu}^{*}\left(\lambda_{1}, \ldots, \lambda_{n}\right)
$$

where $\pi_{\lambda}$ is the irreducible representation of the Lie algebra determined by the partition $\lambda$. We can regard $t_{\mu}^{*}$ as an analogue of the shifted Schur functions, and this is expressed as follows (Lemma-Definition 2.4 in [OO2]):

$$
\begin{aligned}
& t_{\mu}^{* B}\left(\lambda_{1}, \ldots, \lambda_{n}\right)=s_{\mu}\left(\left(\lambda_{1}+n-\frac{1}{2}\right)^{2},\left(\lambda_{2}+n-\frac{3}{2}\right)^{2}, \ldots,\left(\lambda_{n}+\frac{1}{2}\right)^{2} \mid\left(\frac{1}{2}\right)^{2},\left(\frac{3}{2}\right)^{2},\left(\frac{5}{2}\right)^{2}, \ldots\right), \\
& t_{\mu}^{* C}\left(\lambda_{1}, \ldots, \lambda_{n}\right)=s_{\mu}\left(\left(\lambda_{1}+n\right)^{2},\left(\lambda_{2}+n-1\right)^{2}, \ldots,\left(\lambda_{n}+1\right)^{2} \mid 1^{2}, 2^{2}, 3^{2}, \ldots\right), \\
& t_{\mu}^{* D}\left(\lambda_{1}, \ldots, \lambda_{n}\right)=s_{\mu}\left(\left(\lambda_{1}+n-1\right)^{2},\left(\lambda_{2}+n-2\right)^{2}, \ldots,\left(\lambda_{n}+0\right)^{2} \mid 0^{2}, 1^{2}, 2^{2}, \ldots\right) .
\end{aligned}
$$

Here, the superscripts $B, C$, and $D$ indicate the type of the corresponding Lie algebra. Moreover, the right hand sides are the "generalized factorial Schur functions" (see [OO2] for the definition). 
The connection between these functions and $e_{k}^{\Delta_{0}}, e_{k}^{* \Delta_{0}}, h_{k}^{\Delta_{0}}, h_{k}^{* \Delta_{0}}$ is seen from the following relations:

$$
\begin{aligned}
e_{k}^{\Delta^{0}}\left(l_{1}, \ldots, l_{n},-l_{n}, \ldots,-l_{1}\right) & =s_{\left(1^{k}\right)}\left(l_{1}^{2}, \ldots, l_{n}^{2} \mid 0^{2}, 1^{2}, \ldots\right), \\
e_{k}^{* \Delta^{0}}\left(l_{1}, \ldots, l_{n},-l_{n}, \ldots,-l_{1}\right) & =s_{\left(1^{k}\right)}\left(l_{1}^{2}, \ldots, l_{n}^{2} \mid\left(\frac{1}{2}\right)^{2},\left(\frac{3}{2}\right)^{2}, \ldots\right), \\
e_{k}^{* \Delta^{0}}\left(l_{1}, \ldots, l_{n}, 0,-l_{n}, \ldots,-l_{1}\right) & =s_{\left(1^{k}\right)}\left(l_{1}^{2}, \ldots, l_{n}^{2} \mid 1^{2}, 2^{2}, \ldots\right), \\
h_{k}^{* \Delta^{0}}\left(l_{1}, \ldots, l_{n},-l_{n}, \ldots,-l_{1}\right) & =s_{(k)}\left(l_{1}^{2}, \ldots, l_{n}^{2} \mid 1^{2}, 2^{2}, \ldots\right), \\
h_{k}^{\Delta^{0}}\left(l_{1}, \ldots, l_{n},-l_{n}, \ldots,-l_{1}\right) & =s_{(k)}\left(l_{1}^{2}, \ldots, l_{n}^{2} \mid\left(\frac{1}{2}\right)^{2},\left(\frac{3}{2}\right)^{2}, \ldots\right) .
\end{aligned}
$$

These relations themselves follow from Theorems 5.1 5.3. The left hand sides of the second and fourth equalities are also equal to

$$
e_{k}^{\Delta^{0}}\left(l_{1}, \ldots, l_{n}, 0,-l_{n}, \ldots,-l_{1}\right), \quad h_{k}^{\Delta^{0}}\left(l_{1}, \ldots, l_{n}, 0,-l_{n}, \ldots,-l_{1}\right),
$$

respectively (recall Proposition 2.3).

From these relations, we can rewrite Theorem 8.4 in the case of $u=0$ as

$$
\pi_{\lambda}^{\mathfrak{o}_{2 n}}\left(C_{k}^{\mathfrak{o}_{2 n}}(0)\right)=t_{\left(1^{k}\right)}^{* D}\left(\lambda_{1}, \ldots, \lambda_{n}\right), \quad \pi_{\lambda}^{\mathfrak{o}_{2 n+1}}\left(C_{k}^{\mathfrak{o}_{2 n+1}}(0)\right)=t_{\left(1^{k}\right)}^{* B}\left(\lambda_{1}, \ldots, \lambda_{n}\right) .
$$

Similarly, Theorem 8.5 in the case of $u=0$ can be rewritten as

$$
\pi_{\lambda}^{\mathfrak{s p}_{2 n}}\left(D_{k}^{\mathfrak{s p} 2 n}(0)\right)=t_{(k)}^{* C}\left(\lambda_{1}, \ldots, \lambda_{n}\right) .
$$

Combining these with (8.1), we have the following theorem:

Theorem 8.6. We have

$$
C_{k}^{\mathfrak{o}_{2 n}}(0)=\mathbb{T}_{\left(1^{k}\right)}^{D}, \quad C_{k}^{\mathfrak{o}_{2 n+1}}(0)=\mathbb{T}_{\left(1^{k}\right)}^{B}, \quad D_{k}^{\mathfrak{s p}_{N}}(0)=\mathbb{T}_{(k)}^{C} .
$$

Indeed, the eigenvalues of both sides are equal.

This theorem means that we can express $\mathbb{T}_{\left(1^{k}\right)}^{D}, \mathbb{T}_{\left(1^{k}\right)}^{B}$, and $\mathbb{T}_{(k)}^{C}$ in terms of the columndeterminant and the column-permanent. However, an explicit descripsion of $\mathbb{T}_{\mu}$ in terms of a certain noncommutative determinant type function is not given for general $\mu$. Thus the three elements in Theorem [8.6 are lucky exceptions. In [MN], these three elements are studied in more detail, and Capelli type identities for the dual pair $\left(O_{M}, S p_{N}\right)$ are given.

In Theorems 8.4 and 8.5, we expressed the eigenvalues of $C_{k}^{\mathfrak{o}_{N}}(u)$ and $D_{k}^{\mathfrak{s p}_{N}}(u)$ naturally in terms of the functions $e_{k}^{\Delta^{0}}$ and $h_{k}^{* \Delta^{0}}$ for general $u$. This is an advantage of these functions over the function $t_{\mu}^{*}$. Indeed, we cannot describe these eigenvalues in terms of $t_{\mu}^{* B}, t_{\mu}^{* C}$, and $t_{\mu}^{* D}$ so simply. However, it should be noted that, even if $u \neq 0$, we can express the eigenvalue of $C_{k}^{\mathfrak{o}_{N}}(u)$ as a linear combination of $t_{\left(1^{0}\right)}^{* D}, t_{\left(1^{1}\right)}^{* D}, \ldots, t_{\left(1^{k}\right)}^{* D}$ or $t_{\left(1^{0}\right)}^{* B}, t_{\left(1^{1}\right)}^{* B}, \ldots, t_{\left(1^{k}\right)}^{* B}$ using Theorem 4.1. Similarly, the eigenvalue of $D_{k}^{\mathfrak{s p}_{N}}(u)$ can be expressed as a linear combination of $t_{(0)}^{* C}, t_{(1)}^{* C}, \ldots, t_{(k)}^{* C}$ by using Theorem 4.2 .

In the various relations in this article, there was a mysterious duality in the exchanges $s \leftrightarrow s^{*}$ and $\lambda \leftrightarrow \lambda^{\prime}$. It is also mysterious that the functions $e$ and $h^{*}$ played more important roles than $e^{*}$ and $h$ (note that we can rewrite Theorem 8.2 in terms of $h^{*}$ replacing $u$ by $u-1)$. This puzzling phenomenon seems to be related to the following fact: central elements in $U\left(\mathfrak{o}_{N}\right)$ (respectively, $U\left(\mathfrak{s p}_{N}\right)$ ) expressed in terms of the columnpermanent (respectively, the column-determinant) are not known. The author hopes that the theoretical background of these phenomena will become transparent, and the 
Schur type functions in this article will be useful to study the analogues of the quantum immanants in $U\left(\mathfrak{o}_{N}\right)$ and $U\left(\mathfrak{s p}_{N}\right)$ (especially to give their explicit description).

\section{REFERENCES}

[BL] L. C. Biedenharn and J. D. Louck, A new class of symmetric polynomials defined in terms of tableaux, Advances in Appl. Math. 10 (1989), 396-438.

[Ca1] A. Capelli, Über die Zurückführung der Cayley'schen Operation $\Omega$ auf gewöhnliche PolarOperationen, Math. Ann. 29 (1887), 331-338.

[Ca2] Sur les opérations dans la théorie des formes algébriques, Math. Ann. 37 (1890), 1-37.

[CL] W. Y. C. Chen and J. D. Louck, The factorial Schur function, J. Math. Phys. 34 (1993), 41444160.

[HU] R. Howe and T. Umeda, The Capelli identity, the double commutant theorem, and multiplicity-free actions, Math. Ann. 290 (1991), 565-619.

[I1] M. Itoh, Capelli elements for the orthogonal Lie algebras, J. Lie Theory 10 (2000), 463-489.

[I2] _ A Cayley-Hamilton theorem for the skew Capelli elements, J. Algebra 242 (2001), 740761.

[I3] Capelli identities for the dual pair $\left(O_{M}, S p_{N}\right)$, Math. Z. 246 (2004), 125-154.

[I4] Capelli identities for reductive dual pairs, Adv. Math. 194 (2005), 345-397.

[I5] - Two determinants in the universal enveloping algebras of the orthogonal Lie algebras, J. Algebra 314 (2007), 479-506.

[16] _ Two permanents in the universal enveloping algebras of the symplectic Lie algebras, to appear in Internat. J. Math.

[IU] M. Itoh and T. Umeda, On central elements in the universal enveloping algebras of the orthogonal Lie algebras, Compositio Math. 127 (2001), 333-359.

[M1] I. G. Macdonald, Symmetric functions and Hall polynomials, 2nd edition, Oxford Science Publ., 1995.

[M2] Schur functions: themes and variations, in "Séminaire Lotharingien de Combinatoire," Publ. I.R.M.A. Strasbourg 498, 1992, pp. 5-39.

[MN] A. Molev and M. Nazarov, Capelli identities for classical Lie algebras, Math. Ann. 313 (1999), $315-357$.

[MR] R. Mullin and G.-C. Rota, On the Foundations of Combinatorial Theory III: Theory of Binomial Enumeration, in "Graph Theory and Its Applications," edited by Bernard Harris, Academic Press, New York, 1970, 167-213.

[N] M. Nazarov, Quantum Berezinian and the classical Capelli identity, Lett. Math. Phys. 21 (1991), $123-131$.

[O] A. Okounkov, Quantum immanants and higher Capelli identities, Transform. Groups 1 (1996), no. $1,99-126$.

[OO1] A. Okounkov and G. Olshanski, Shifted Schur functions, Algebra i Analiz 9 (1997), No. 2 (Russian); English version in St. Petersburg Math. J. 9 (1998), 239-300.

[OO2] - Shifted Schur functions II. Binomial formula for characters of classical groups and applications, in "A.A.Kirillov Seminar on Representation Theory," Amer. Math. Soc. Translations (2) Vol. 181, (1998), pp. 245-271.

[R] Steven Roman, The Umbral Calculus, Academic Press, New York, 1984; republication: Dover Publications, 2005.

[RKO] G.-C. Rota, D. Kahaner, and A. Odlyzko, Finite Operator Calculus, Journal of Mathematical Analysis and its Applications 42, no. 3 (1973), 684-760.

[S] R.P. Stanley, Enumerative combinatorics. Vol. 2. Cambridge Studies in Advanced Mathematics, 62. Cambridge University Press, Cambridge, 1999.

[U1] T. Umeda, The Capelli identities, a century after, Sūgaku 46 (1994), 206-227, (in Japanese); English transl. in "Selected Papers on Harmonic Analysis, Groups, and Invariants," AMS Translations, Series 2, vol. 183 (1998), pp. 51-78, ed. by K. Nomizu.

[U2] — Newton's formula for $\mathfrak{g l}_{n}$, Proc. Amer. Math. Soc. 126 (1998), 3169-3175. 
[U3] _ On Turnbull identity for skew symmetric matrices, Proc. Edinburgh Math. Soc. (2) 43 (2000), 379-393.

[U4] Application of Koszul complex to Wronski relations for $U\left(\mathfrak{g l}_{n}\right)$, Comment. Math. Helv. 78 (2003), 663-680.

[U5] - On the proof of the Capelli identities, Funkcialaj Ekvacioj 51 (2008), 1-15.

[W] A. Wachi, Central elements in the universal enveloping algebras for the split realization of the orthogonal Lie algebras, Lett. Math. Phys. 77 (2006), 155-168.

Department of Mathematics and Computer Science, Faculty of Science, Kagoshima UNIVERSITY, KAGOSHIMA 890-0065, JAPAN

E-mail address: itoh@sci.kagoshima-u.ac.jp 